\title{
Further studies of the photoproduction of isolated photons with a jet at HERA
}

\section{The ZEUS collaboration}

\author{
E-mail: m.wing@ucl.ac.uk
}

ABSTRACT: In this extended analysis using the ZEUS detector at HERA, the photoproduction of isolated photons together with a jet is measured for different ranges of the fractional photon energy, $x_{\gamma}^{\text {meas }}$, contributing to the photon-jet final state. Cross sections are evaluated in the photon transverse-energy and pseudorapidity ranges $6<E_{T}^{\gamma}<15 \mathrm{GeV}$ and $-0.7<\eta^{\gamma}<0.9$, and for jet transverse-energy and pseudorapidity ranges $4<E_{T}^{\text {jet }}<$ $35 \mathrm{GeV}$ and $-1.5<\eta^{\text {jet }}<1.8$, for an integrated luminosity of $374 \mathrm{pb}^{-1}$. The kinematic observables studied comprise the transverse energy and pseudorapidity of the photon and the jet, the azimuthal difference between them, the fraction of proton energy taking part in the interaction, and the difference between the pseudorapidities of the photon and the jet. Higher-order theoretical calculations are compared to the results.

KEYwords: Lepton-Nucleon Scattering, QCD, Photon production

ARXIV EPRINT: 1405.7127 


\section{Contents}

1 Introduction 1

2 Experimental set-up $\quad 3$

3 Theoretical models $\quad 4$

4 Monte Carlo event simulation 5

5 Event selection and reconstruction 5

$\begin{array}{lll}6 & \text { Extraction of the photon signal } & 7\end{array}$

$\begin{array}{llr}7 & \text { Systematic uncertainties } & 8\end{array}$

$\begin{array}{llr}8 & \text { Results } & 9\end{array}$

9 Conclusions $\quad 11$

$\begin{array}{ll}\text { The ZEUS collaboration } & 24\end{array}$

\section{Introduction}

In a recently published paper [1], the ZEUS collaboration presented cross sections for events containing an isolated high-energy photon, with and without a jet, produced in photoproduction at the HERA collider using the full HERA II data set. Such events can provide a direct probe of the underlying partonic process in high-energy collisions involving photons, since the emission of a high-energy photon is largely unaffected by parton hadronisation. In photoproduction processes in $e p$ collisions at HERA, the exchanged virtual photon is quasi-real, with small virtuality, $Q^{2}$, conventionally required to be less than $1 \mathrm{GeV}^{2}$. These measurements follow earlier analyses of isolated photons in photoproduction by the ZEUS and H1 collaborations [2-7], as well as in deep inelastic scattering (DIS) [8-11]. In the analysis presented here, the most recent ZEUS photoproduction measurements are extended, using the same data as used previously.

In "direct" production processes, the entire incoming photon is absorbed by an outgoing quark from the incoming proton, while in "resolved" processes, the photon's hadronic structure provides a quark or gluon that interacts with a parton from the proton. Figure 1 gives examples of the lowest-order (LO) direct and resolved diagrams for high-energy photoproduction of photons in quantum chromodynamics (QCD). ${ }^{1}$ Higher-order processes

\footnotetext{
${ }^{1}$ Photons that are radiated in the hard scattering process, rather than resulting from meson decay, are commonly called "prompt". An alternative nomenclature is to refer to such photons as "direct"; thus figures 1(a) and 1(b) would be called "direct-direct" and "resolved-direct" diagrams, respectively.
} 


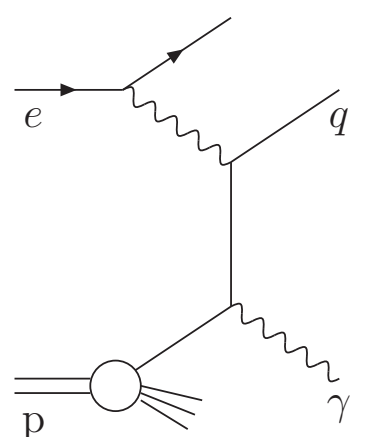

(a)

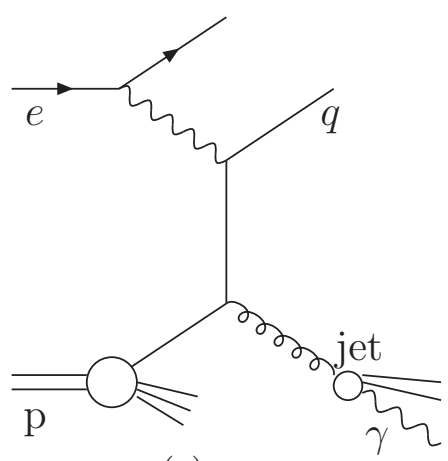

(c)

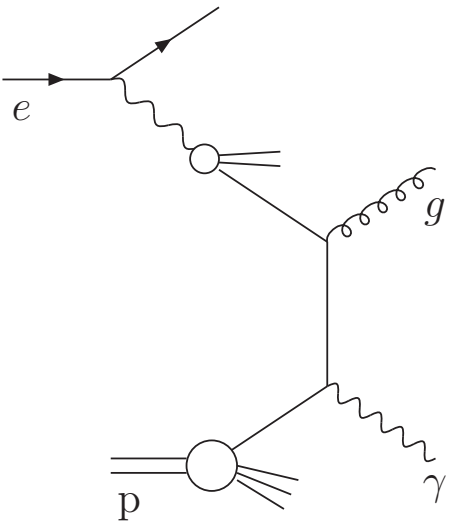

(b)

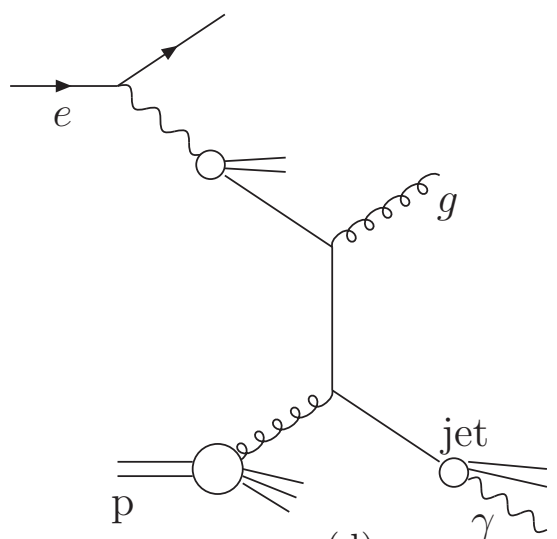

(d)

Figure 1. Examples of (a) direct-prompt and (b) resolved-prompt processes at leading order in $\mathrm{QCD}$, and the related (c) direct and (d) resolved fragmentation processes.

also include "fragmentation processes" in which a photon is radiated within a jet, also illustrated in figure 1. Such processes are suppressed by requiring that the outgoing photon must be isolated.

Resolved and direct processes may be partially distinguished in events containing a high- $E_{T}$ photon and a jet by means of the quantity

$$
x_{\gamma}^{\text {meas }}=\frac{E^{\gamma}+E^{\mathrm{jet}}-p_{Z}^{\gamma}-p_{Z}^{\mathrm{jet}}}{E^{\text {all }}-p_{Z}^{\text {all }}}
$$

which measures the fraction of the incoming photon energy that is given to the photon and the jet. The quantities $E^{\gamma}$ and $E^{\text {jet }}$ denote the energies of the photon and the jet, respectively, $p_{Z}$ denotes the corresponding longitudinal momenta, ${ }^{2}$ and the suffix "all"

\footnotetext{
${ }^{2}$ The ZEUS coordinate system is a right-handed Cartesian system, with the $Z$ axis pointing in the nominal proton beam direction, referred to as the "forward direction", and the $X$ axis pointing towards the centre of HERA. The coordinate origin is at the centre of the central tracking detector. The pseudorapidity is defined as $\eta=-\ln \left(\tan \frac{\theta}{2}\right)$, where the polar angle, $\theta$, is measured with respect to the $Z$ axis.
} 
refers to all the measured final-state particles of an event. At LO, $x_{\gamma}^{\text {meas }}=1$ for direct events, while any value in the range $(0,1)$ may be taken for resolved events. At higher order, the first statement no longer precisely holds, but the presence of direct processes generates a prominent peak in the cross section at high $x_{\gamma}^{\text {meas }}$. Here, measurements in a direct-dominated region are presented by selecting events with $x_{\gamma}^{\text {meas }}>0.8$, and in a resolved-dominated region by selecting events with $x_{\gamma}^{\text {meas }}<0.8$. This enables the behaviour of the photoproduction process to be explored in more detail.

Several kinematic quantities are also measured beyond those presented previously. The quantity

$$
x_{p}^{\mathrm{obs}}=\left(E_{T}^{\gamma} \exp \eta^{\gamma}+E_{T}^{\mathrm{jet}} \exp \eta^{\mathrm{jet}}\right) / 2 E_{p}
$$

estimates the fraction of proton energy taken by the parton that interacts with the photon; its distribution is sensitive to the proton's partonic structure. Here, $E_{T}$ denotes transverse energy, $\eta$ denotes pseudorapidity, and $E_{p}$ is the energy of the proton beam. The difference in pseudorapidities, $\eta^{\gamma}-\eta^{\text {jet}}$, is sensitive to the dynamical details of the hard scattering process, in particular to the spin of the exchanged quantum [12]. The quantity $\Delta \phi$, defined as the absolute difference between the azimuths of the photon and the high- $E_{T}$ jet, is sensitive to the presence of higher-order gluon radiation in the event, especially relative to the outgoing quark, which can generate non-collinearity between the photon and the leading jet. All three of these quantities are insensitive to Lorentz boosts along the $Z$ axis.

Predictions from QCD-based models are compared to the measurements. The cross sections for isolated-photon production in photoproduction have been calculated to nextto-leading order (NLO) by Fontannaz, Guillet and Heinrich (FGH) [13-15]. Calculations based on the $k_{T}$-factorisation approach have been made by Lipatov, Malyshev and Zotov (LMZ) [16-19].

\section{Experimental set-up}

The measurements are based on a data sample corresponding to an integrated luminosity of $374 \pm 7 \mathrm{pb}^{-1}$, taken during the years 2004 to 2007 with the ZEUS detector at HERA. During this period, HERA ran with an electron or positron beam energy of $27.5 \mathrm{GeV}$ and a proton beam energy of $E_{p}=920 \mathrm{GeV}$. The sample is a sum of $e^{+} p$ and $e^{-} p$ data. $^{3}$

A detailed description of the ZEUS detector can be found elsewhere [20]. Charged particles were measured in the central tracking detector (CTD) [21-23] and a silicon micro vertex detector (MVD) [24] which operated in a magnetic field of $1.43 \mathrm{~T}$ provided by a thin superconducting solenoid. The high-resolution uranium-scintillator calorimeter (CAL) [25-28] consisted of three parts: the forward (FCAL), the barrel (BCAL) and the rear (RCAL) calorimeters. The BCAL covered the pseudorapidity range -0.74 to 1.01 as seen from the nominal interaction point, and the FCAL and RCAL extended the coverage to the range -3.5 to 4.0. Each part of the CAL was subdivided into elements referred to as cells. The barrel electromagnetic calorimeter (BEMC) cells had a pointing geometry aimed

\footnotetext{
${ }^{3}$ Hereafter "electron" refers to both electrons and positrons unless otherwise stated.
} 
at the nominal interaction point, with a cross section approximately $5 \times 20 \mathrm{~cm}^{2}$, with the finer granularity in the $Z$ direction and the coarser in the $(X, Y)$ plane. This fine granularity allows the use of shower-shape distributions to distinguish isolated photons from the products of neutral meson decays such as $\pi^{0} \rightarrow \gamma \gamma$. The CAL energy resolution, as measured under test-beam conditions, was $\sigma(E) / E=0.18 / \sqrt{E}$ for electrons and $0.35 / \sqrt{E}$ for hadrons, where $E$ is in GeV.

The luminosity was measured [29] using the Bethe-Heitler reaction $e p \rightarrow e \gamma p$ by a luminosity detector which consisted of two independent systems: a lead-scintillator calorimeter [30-32] and a magnetic spectrometer [33].

\section{Theoretical models}

Two theoretical models are considered. In the approach of FGH $[13,14]$, the LO and NLO diagrams and the box-diagram term are calculated explicitly. Fragmentation processes are calculated in terms of a fragmentation function in which a quark or gluon gives rise to a photon; an experimentally determined non-perturbative parameterisation is used as input to the theoretical calculation [34]. Fragmentation and box terms each contribute about $10 \%$ to the total cross section. The CTEQ6 [35] and AFG04 [36] parton densities are used for the proton and photon, respectively. Theoretical uncertainties arise due to the choice of renormalisation, factorisation and fragmentation scales. They were estimated, using a more conservative approach [15] than in the original published paper [13], by varying the renormalisation scale by factors of 0.5 and 2.0 , since this gave the largest effect on the cross sections.

The $k_{T}$-factorisation method used by LMZ [16-18] makes use of unintegrated parton densities in the proton, using the KMR formalism [37, 38] based on the MSTW08 proton parton densities [39]. In addition to the hard QCD subprocess, the model incorporates a parton evolution cascade, one jet from which can be taken as the leading jet in the analysis. Fragmentation terms and the quark content of the resolved photon are not included, but the box diagram is included together with $2 \rightarrow 3$ subprocesses to represent the LO direct and resolved photon contributions. The calculation used in the previous ZEUS analysis [1] has been augmented by a term that takes account of the gluon content of the resolved photon, and further technical changes have been implemented [19]. Uncertainties associated with the hard scale were provided by the authors. There is a further overall statistical uncertainty on the set of results for each variable, of the order of $10 \%$ for the results presented here.

All results are presented at the hadron level; to make use of the theoretical predictions, cuts equivalent to the experimental kinematic selections including the photon isolation (see section 5) were applied at the parton level. Hadronisation corrections were then evaluated (section 4) and applied to the theoretical calculations to enable a comparison to the experimental data. 


\section{Monte Carlo event simulation}

Monte Carlo (MC) event samples were employed to evaluate the detector acceptance and event-reconstruction efficiency, and to provide signal and background distributions. The program Pyтhia 6.416 [40] was used to generate the direct and resolved prompt-photon processes at LO, and also $2 \rightarrow 2$ parton-parton scattering processes not involving photons ("dijet events"), making use of the CTEQ4 [41] and GRV [42, 43] proton and photon parton densities. The program was run using the default parameters with minor modifications. ${ }^{4}$ The isolated photons measured in the experiment are accompanied by backgrounds from neutral mesons in hadronic jets, in particular $\pi^{0}$ and $\eta$, where the meson decay products create an energy cluster in the BCAL that passes the selection criteria for a photon. The dijet event samples included background events of this kind which were extracted for use in the analysis. The PyтніA dijet events in which a high-energy photon was radiated from a quark or lepton ("radiative events") were not used in the background samples but were defined, in accordance with theory, as a component of the signal.

Event samples were also generated using the HERWIG 6.510 program [44], again with minor modifications to the default parameters. The PYTHIA and HeRwig programs differ significantly in their treatment of parton showers, and in the use of a string-based hadronisation scheme in Pythia but a cluster-based scheme in HeRwig.

The generated MC events were passed through the ZEUS detector and trigger simulation programs based on GEANT 3.21 [45]. They were then reconstructed and analysed using the same programs as used for the data. The hadronisation corrections to the theory calculations were evaluated using Pythia and HeRwig, the two programs being in agreement to a few percent; Pythia was used to provide the values for the present analysis. No uncertainties were applied to these corrections. They were calculated by running the same jet algorithm and event selections, including the isolation criterion, on the generated partons and on the hadronised final state in the direct and resolved prompt-photon MC events.

\section{Event selection and reconstruction}

The basic event selection and reconstruction was performed as previously. A three-level trigger system was used to select events online [20, 46, 47]:

- the first-level trigger required a loosely measured track in the CTD and a minimum energy deposited in the CAL;

- at the second level, the event conditions were tightened;

- at the third level, the event was reconstructed and a high-energy photon candidate was required. Most deep inelastic scattering events were rejected.

\footnotetext{
${ }^{4}$ In particular, the PyTHIA parameter PARP(67) was set to 4.0 and multiple parton interactions were turned off. In HERWIG the parameters ISPAC, QSPAC, and PTRMS were set to 2, 4.0, and 0.44.
} 
In the offline event analysis, some general conditions were applied as follows:

- to reduce background from non-ep collisions, events were required to have a reconstructed vertex position, $Z_{\mathrm{vtx}}$, within the range $\left|Z_{\mathrm{vtx}}\right|<40 \mathrm{~cm}$;

- to remove any DIS contamination, no scattered beam electron was permitted in the ZEUS detector;

- a range of incoming virtual photon energies was selected by the requirement $0.2<$ $y_{\mathrm{JB}}<0.7$, where $y_{\mathrm{JB}}=\sum_{i} E_{i}\left(1-\cos \theta_{i}\right) / 2 E_{e}$ and $E_{e}$ is the energy of the electron beam. Here, $E_{i}$ is the energy of the $i$-th CAL cell, $\theta_{i}$ is its polar angle and the sum runs over all cells [48]. The lower cut strengthened the trigger requirements and the upper cut further suppressed remaining deep inelastic scattering events.

The subsequent event analysis made use of energy-flow objects (EFOs) [49, 50], which were constructed from clusters of calorimeter cells, associated with tracks when appropriate. Tracks not associated with calorimeter clusters were also used. Photon candidates were EFOs with no associated track and with at least $90 \%$ of the reconstructed energy measured in the BEMC. Candidate EFOs with wider electromagnetic showers than are typical for a single photon were accepted, in order to evaluate the backgrounds.

Jet reconstruction was performed, making use of all the EFOs in the event including photon candidates, by means of the $k_{T}$ clustering algorithm [51] in the $E$-scheme in the longitudinally invariant inclusive mode [52] with the radius parameter set to 1.0. By construction, one of the jets found by this procedure corresponds to or includes the photon candidate. An additional accompanying jet was required; if more than one was found in the designated angular range, that with the highest transverse energy, $E_{T}^{\text {jet }}$, was used. In the kinematic region used, the resolution of the jet transverse energy was about $15-20 \%$, estimated using MC simulations.

To reduce the fragmentation contribution and the background from the decay of neutral mesons within jets, the photon candidate was required to be isolated from other hadronic activity. This was imposed by requiring that the photon-candidate EFO had at least $90 \%$ of the total energy of the reconstructed jet of which it formed a part. High- $E_{T}$ photons radiated from scattered leptons were further suppressed by rejecting photons with a nearby track. This was achieved by demanding $\Delta R>0.2$, where $\Delta R=\sqrt{(\Delta \phi)^{2}+(\Delta \eta)^{2}}$ is the distance to the nearest reconstructed track with momentum greater than $250 \mathrm{MeV}$ in the $\eta-\phi$ plane, where $\phi$ is the azimuthal angle. This latter condition was applied only at the detector level, and not in the hadron- or parton-level calculations.

Events were finally selected with the following kinematic conditions:

- each event was required to contain an isolated photon candidate with a reconstructed transverse energy, $E_{T}^{\gamma}$, in the range $6<E_{T}^{\gamma}<15 \mathrm{GeV}$ and with pseudorapidity, $\eta^{\gamma}$, in the range $-0.7<\eta^{\gamma}<0.9$;

- a hadronic jet was required with $E_{T}^{\text {jet }}$ between 4 and $35 \mathrm{GeV}$ and lying within the pseudorapidity, $\eta^{\text {jet }}$, range $-1.5<\eta^{\text {jet }}<1.8$; 
- selections were made for all $x_{\gamma}^{\text {meas }}$, giving a total of 12450 events, and for $x_{\gamma}^{\text {meas }}>0.8$ and $x_{\gamma}^{\text {meas }}<0.8$. The latter two conditions selected events in direct-enhanced and resolved-enhanced regions, respectively. An additional selection was made with events having $x_{\gamma}^{\text {meas }}<0.7$.

\section{Extraction of the photon signal}

The selected samples contain a large admixture of background events in which one or more neutral mesons, such as $\pi^{0}$ and $\eta$, have decayed to photons, thereby producing a photon candidate in the BEMC. The photon signal was extracted statistically following the approach used in previous ZEUS analyses [1, 8-11]. The method made use of the energyweighted width, measured in the $Z$ direction, of the BEMC energy-cluster comprising the photon candidate. This width was calculated as

$$
\langle\delta Z\rangle=\sum_{i} E_{i}\left|Z_{i}-Z_{\text {cluster }}\right| /\left(w_{\text {cell }} \sum_{i} E_{i}\right),
$$

where $Z_{i}$ is the $Z$ position of the centre of the $i$-th cell, $Z_{\text {cluster }}$ is the energy-weighted centroid of the EFO cluster, $w_{\text {cell }}$ is the width of the cell in the $Z$ direction, and $E_{i}$ is the energy recorded in the cell. The sum runs over all BEMC cells in the EFO.

The number of isolated-photon events in the data was determined by a $\chi^{2}$ fit to the $\langle\delta Z\rangle$ distribution in the range $0.05<\langle\delta Z\rangle<0.8$, varying the relative fractions of the signal and background components as represented by histogram templates obtained from the MC. The fit was performed for each measured cross-section bin, with $\chi^{2}$ values of typically 1.1 per degree of freedom (e.g. 31/28), verifying that the signal and background were well understood. The extracted signals corresponded overall to $6262 \pm 132$ events with a photon and an accompanying jet. A set of typical fits for different ranges of the photon transverse energy is shown in figure 2 and illustrates how the signal-to-background ratio improves with increasing $E_{T}^{\gamma}$.

A bin-by-bin correction method was used to determine the production cross section in a given variable, by means of the relationship

$$
\frac{d \sigma}{d Y}=\frac{\mathcal{A} N(\gamma)}{\mathcal{L} \Delta Y},
$$

where $N(\gamma)$ is the number of photons in a bin as extracted from the fit, $\Delta Y$ is the bin width, $\mathcal{L}$ is the total integrated luminosity, and $\mathcal{A}$ is the acceptance correction. The acceptance correction was calculated, using $\mathrm{MC}$ samples, as the ratio of the number of events that were generated in the given bin to the number of events obtained in the bin after event reconstruction. Its value was typically 1.2.

Allowance must be made for the different acceptances for the direct and the resolved processes, as modelled by PyтHIA. Over the entire $x_{\gamma}^{\text {meas }}$ range, a reasonable phenomenological description of the data can be obtained using a MC sample consisting of a 50:40 mixture of PYTHIA-simulated direct and resolved events, normalised to the data, with a $10 \%$ admixture of radiative events divided equally between direct and resolved. The acceptance factors were calculated using this model, applying selections for the chosen $x_{\gamma}^{\text {meas }}$ 


\section{ZEUS}
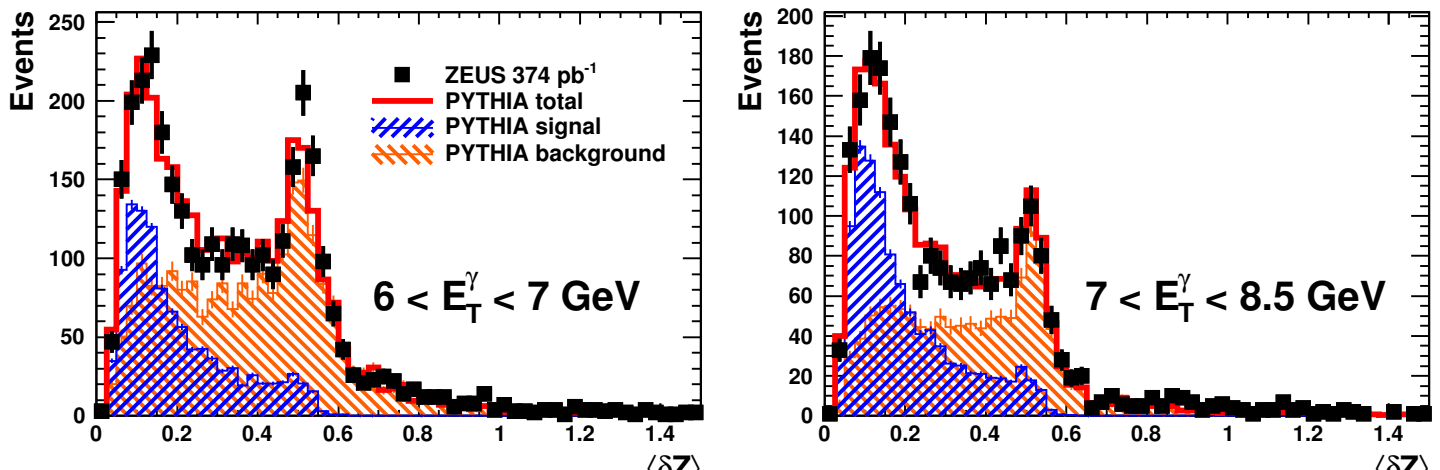

$\langle\delta \mathbf{Z}\rangle$
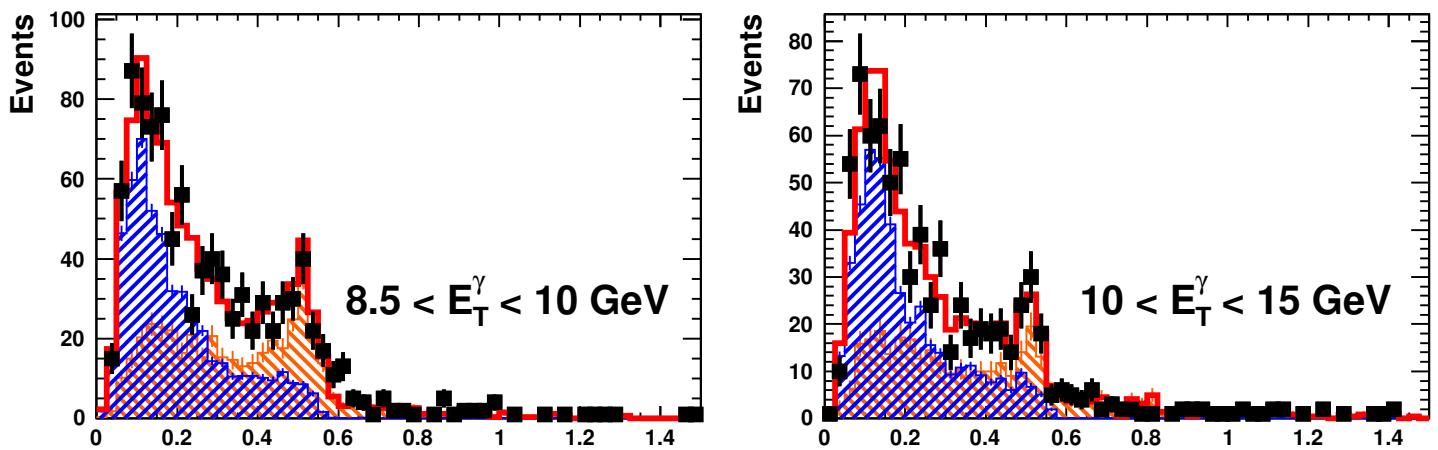

$\langle\delta \mathbf{Z}\rangle$

Figure 2. Examples of fits to $\langle\delta Z\rangle$ for different ranges of the photon transverse energy, showing the signal and background contributions and the fitted total.

regions. Small corrections were applied for the trigger efficiency modelling and a residual contamination by DIS events [1].

The photon energy scale was calibrated by means of an analysis of deeply virtual Compton scattering events recorded by ZEUS, in which the detected final-state particles comprised a scattered electron, whose energy measurement is well understood, and a balancing outgoing photon.

\section{Systematic uncertainties}

The most significant sources of systematic uncertainty were evaluated as follows:

- to allow for uncertainties in the simulation of the hadronic final state, the cross sections were recalculated using HERWIG to model the signal and background events. The ensuing changes in the results correspond to an uncertainty of typically up to $8 \%$, but rising to $18 \%$ in the highest bin of $x_{\gamma}^{\text {meas; }}$

- the energy of the photon candidate was varied by $\pm 2 \%$ in the $\mathrm{MC}$ at the detector level. Independently, the energy of the accompanying jet was varied by an amount decreasing from $\pm 4.5 \%$ to $\pm 2.5 \%$ as $E_{T}^{\text {jet }}$ increases from $4 \mathrm{GeV}$ to above $10 \mathrm{GeV}$. Each of these gave variations in the measured cross sections of typically $5 \%$. 
Further systematic uncertainties were evaluated as follows:

- the uncertainty in the acceptance due to the estimation of the relative fractions of direct and resolved events and radiative events in the MC sample was estimated by varying these fractions by $\pm 15 \%$ and $\pm 5 \%$ respectively in absolute terms; the changes in the cross sections were typically $\pm 2 \%$ in each case;

- the dependence of the result on the modelling of the hadronic background by the MC was investigated by varying the upper limit for the $\langle\delta Z\rangle$ fit in the range [0.6, 1.0]; this gave a $\pm 2 \%$ variation;

- the $E_{T}^{\gamma}, \eta^{\text {jet }}$ and $\Delta \phi$ distributions in the MC were reweighted simultaneously to provide a closer agreement with the data, and the cross sections were re-evaluated. This generated changes of typically $\pm 2 \%$.

Other sources of systematic uncertainty were found to be negligible. These included the modelling of the track-isolation cut and the track-momentum cut, and also the cuts on photon isolation, the electromagnetic fraction of the photon shower, $y_{\mathrm{JB}}$ and $Z_{\mathrm{vtx}}$. Except for the uncertainty on the modelling of the hadronic final state, the major uncertainties were treated as symmetric, and all the uncertainties were combined in quadrature. The uncertainties of $2.0 \%$ on the trigger efficiency and $1.9 \%$ on the luminosity measurement were not included in the tables and figures.

\section{Results}

Differential cross sections were calculated for the production of an isolated photon with at least one accompanying jet, in the kinematic region defined by $Q^{2}<1 \mathrm{GeV}^{2}, 0.2<y<0.7$, $-0.7<\eta^{\gamma}<0.9,6<E_{T}^{\gamma}<15 \mathrm{GeV}, 4<E_{T}^{\text {jet }}<35 \mathrm{GeV}$ and $-1.5<\eta^{\text {jet }}<1.8$. All quantities were evaluated at the hadron level in the laboratory frame, and $y$ is defined as the fraction of the incoming lepton energy that is given to the virtual photon. The jets were formed according to the $k_{T}$ clustering algorithm with the radius parameter set to 1.0. Photon isolation was imposed such that at least $90 \%$ of the energy of the jet-like object containing the photon originated from the photon. If more than one accompanying jet was found within the designated $\eta^{\text {jet }}$ range in an event, that with highest $E_{T}^{\text {jet }}$ was taken. Cross sections in $E_{T}^{\text {jet }}$ above $15 \mathrm{GeV}$ are omitted from the tables and figure 4 owing to limited statistics, but this kinematic region is included in the other cross-section measurements.

Complementing the previously published cross sections [1] for the entire $x_{\gamma}^{\text {meas }}$ range, differential cross sections as functions of $E_{T}^{\gamma}, \eta^{\gamma}, E_{T}^{\text {jet }}$ and $\eta^{\text {jet }}$ are shown in figures 3-4. Here the selections of $x_{\gamma}^{\text {meas }}>0.8$ and $x_{\gamma}^{\text {meas }}<0.8$ have been applied to define ranges that enhance the direct and resolved processes. In the PYTHIA model that was used, the upper and lower $x_{\gamma}^{\text {meas }}$ ranges contain direct and resolved events in the ratios 86:14 and 22:78, respectively.

To within the theoretical uncertainties, the cross section predicted by FGH is in quantitative agreement with the data; the LMZ predicted cross section also agrees well for the photon and $E_{T}^{\text {jet }}$ variables, but it is in disagreement with the $\eta^{\text {jet }}$ distribution for 


\section{ZEUS}
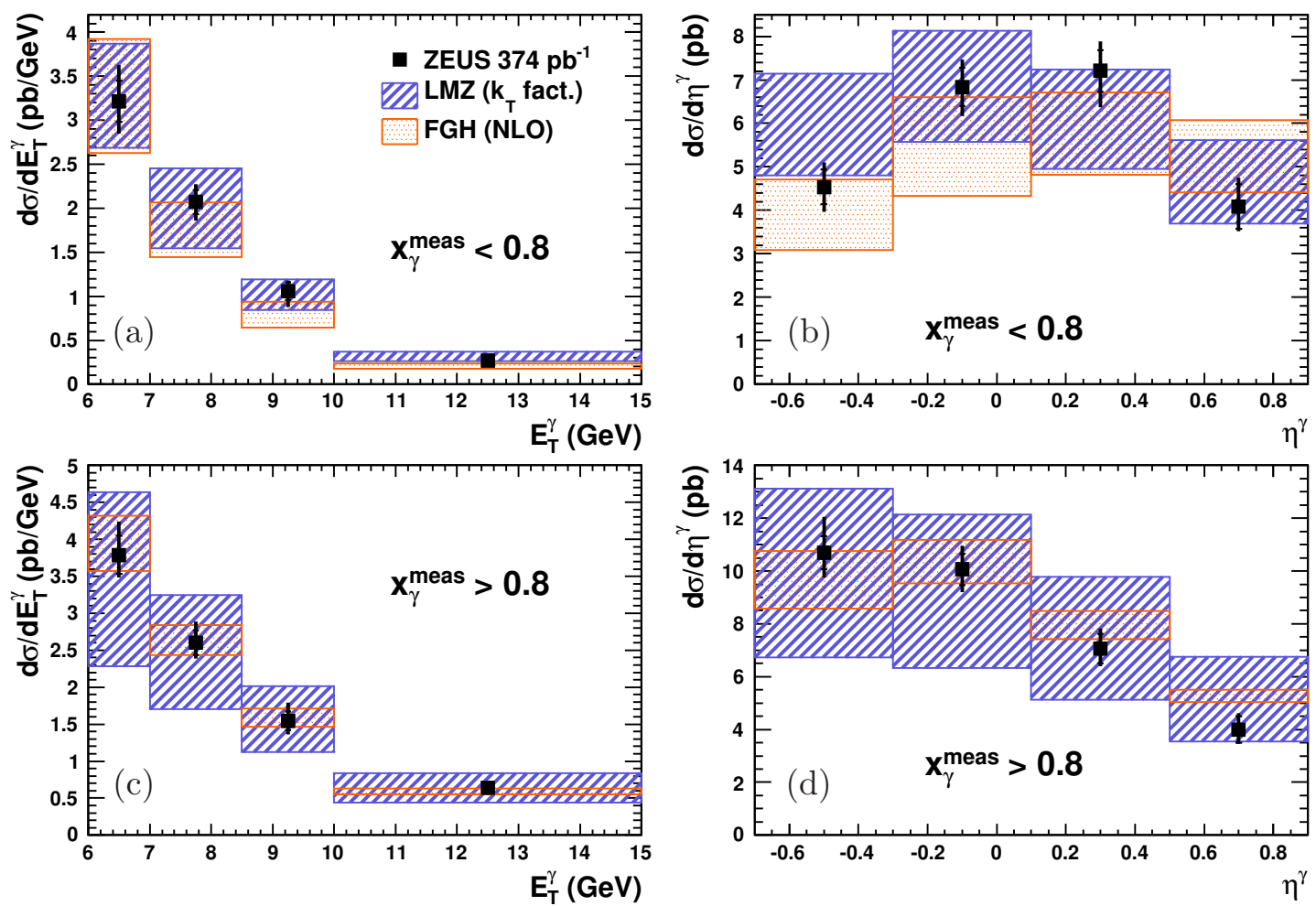

Figure 3. Differential cross sections as functions of $(\mathrm{a}, \mathrm{c}) E_{T}^{\gamma}$ and $(\mathrm{b}, \mathrm{d}) \eta^{\gamma}$ in different ranges of $x_{\gamma}^{\text {meas }}$, for events containing an isolated photon accompanied by a jet, compared to predictions from FGH and LMZ. The kinematic region of the measurement is described in the text. The inner and outer error bars respectively denote statistical uncertainties and statistical uncertainties combined with systematic uncertainties in quadrature. The theoretical uncertainties are shown as hatched and dotted bands.

$x_{\gamma}^{\text {meas }}<0.8$. This disagreement may be due to the modelling of the jet from the parton cascade in the present version of the LMZ model.

The variables $x_{p}^{\text {obs }}$ and $\eta^{\gamma}-\eta^{\text {jet}}$, presented in figures 5 and 6 , also include results evaluated for the entire $x_{\gamma}^{\text {meas }}$ range. They are well described by FGH but slightly less so by LMZ.

Differential cross sections as functions of $\Delta \phi$ are shown in figure 7 . The data are compared to FGH and LMZ, with similar conclusions as before, and are also compared to the versions of Pythia and Herwig described in section 4. The MC programs both give a reasonable description of the data. These results demonstrate that parton showers used in conjunction with LO MC programs can give a good description of higher-order contributions, as also observed in other reactions [53-55].

Tables 1 to 6 give the numerical values of the above results, together with the hadronisation factors that were applied to the theory. For further information, cross sections calculated in the range $x_{\gamma}^{\text {meas }}<0.7$ are also listed. These have a direct:resolved ratio of 15:85 as modelled by PүтніA and show features that are similar to the plotted results. 


\section{ZEUS}
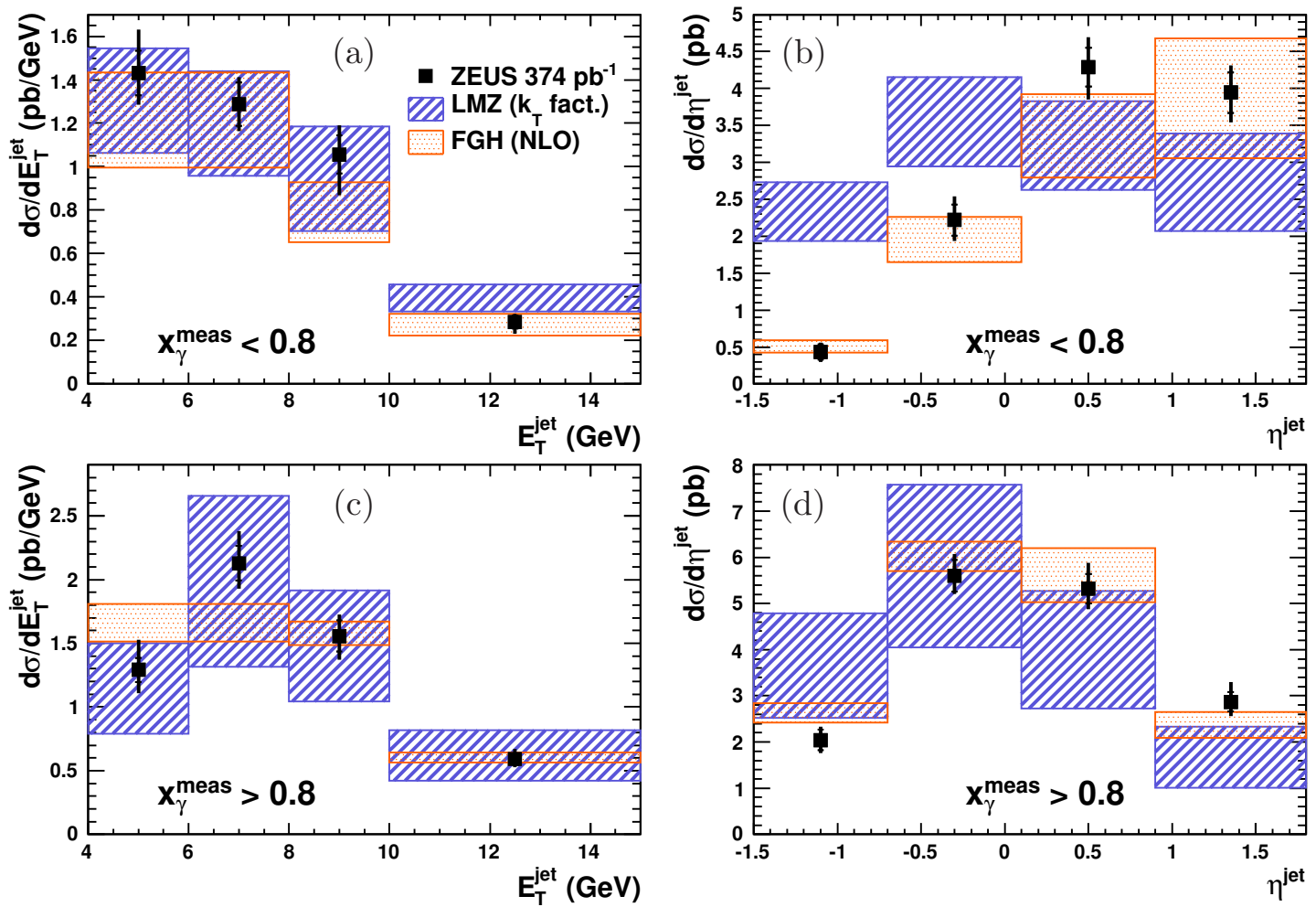

Figure 4. Differential cross sections as functions of (a, c) $E_{T}^{\text {jet }}$ and (b, d) $\eta^{\text {jet }}$, for events containing an isolated photon accompanied by a jet, compared to predictions from FGH and LMZ. The first two FGH points in (a, c) have been averaged into a single bin for calculational reasons. Other details as for figure 3 .

\section{Conclusions}

The production of isolated photons with an accompanying jet has been measured in photoproduction with the ZEUS detector at HERA using an integrated luminosity of $374 \pm 7 \mathrm{pb}^{-1}$. The present measurements extend earlier ZEUS results. Differential cross sections are presented in a kinematic region defined in the laboratory frame by: $Q^{2}<1$ $\mathrm{GeV}^{2}, 0.2<y<0.7,-0.7<\eta^{\gamma}<0.9,6<E_{T}^{\gamma}<15 \mathrm{GeV}, 4<E_{T}^{\mathrm{jet}}<35 \mathrm{GeV}$ and $-1.5<\eta^{\text {jet }}<1.8$. Photon isolation was imposed such that at least $90 \%$ of the energy of the jet-like object containing the photon originated from the photon.

Differential cross sections are given in terms of the transverse energy and pseudorapidity of the photon and the jet, and in terms of $x_{p}^{\text {obs }}, \eta^{\gamma}-\eta^{\text {jet }}$ and $\Delta \phi$ in high and low regions of $x_{\gamma}^{\text {meas }}$. The latter three variables are also presented for the entire observed $x_{\gamma}^{\text {meas }}$ range. The NLO-based predictions of Fontannaz, Guillet and Heinrich reproduce all the measured experimental distributions well. The $k_{T}$-factorisation approach of Lipatov, Malyshev and Zotov describes most of the distributions well, with the exception of the jet pseudorapidity at low $x_{\gamma}^{\text {meas }}$. 
ZEUS
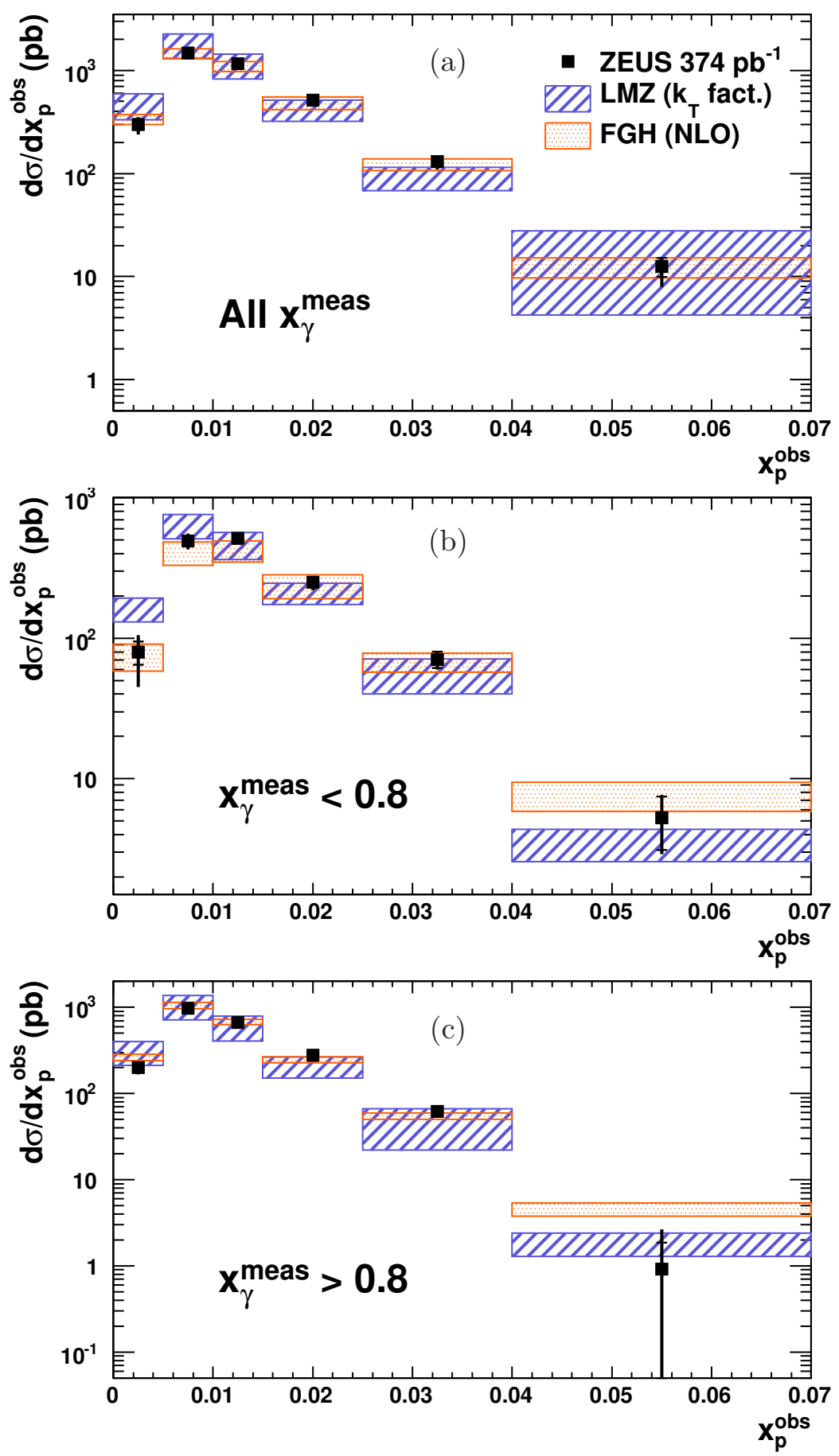

Figure 5. Differential cross sections as functions of $x_{p}^{\text {obs }}$ for (a) all $x_{\gamma}^{\text {meas }}$, (b) $x_{\gamma}^{\text {meas }}>0.8$ (c) $x_{\gamma}^{\text {meas }}<0.8$ for events containing an isolated photon accompanied by a jet, compared to predictions from FGH and LMZ. Other details as for figure 3. 


\section{ZEUS}
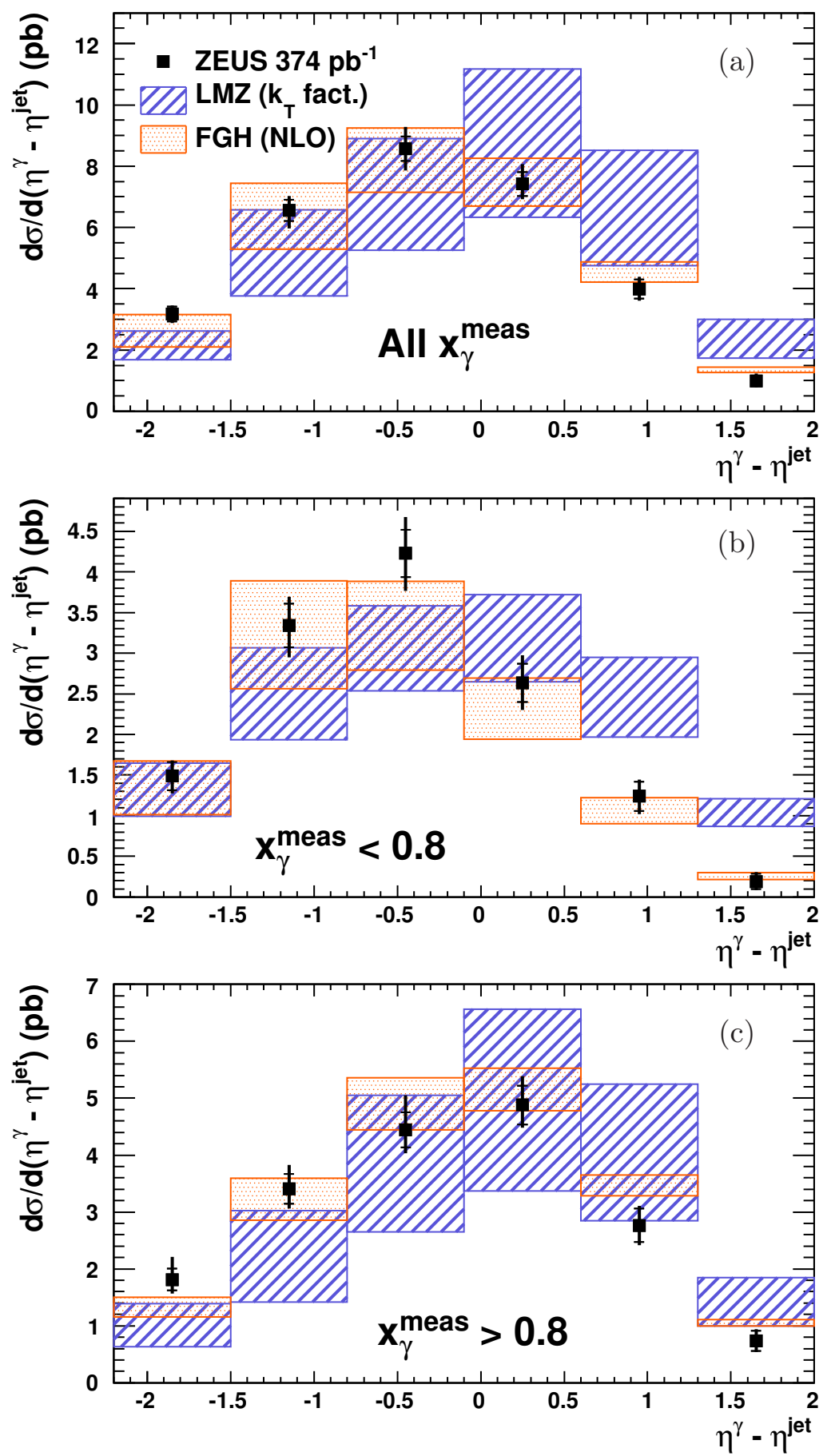

Figure 6. Differential cross sections as functions of $\eta^{\gamma}-\eta^{\text {jet }}$ for (a) all $x_{\gamma}^{\text {meas }}$, (b) $x_{\gamma}^{\text {meas }}>0.8$ (c) $x_{\gamma}^{\text {meas }}<0.8$ for events containing an isolated photon accompanied by a jet, compared to predictions from FGH and LMZ. Other details as for figure 3. 


\section{ZEUS}
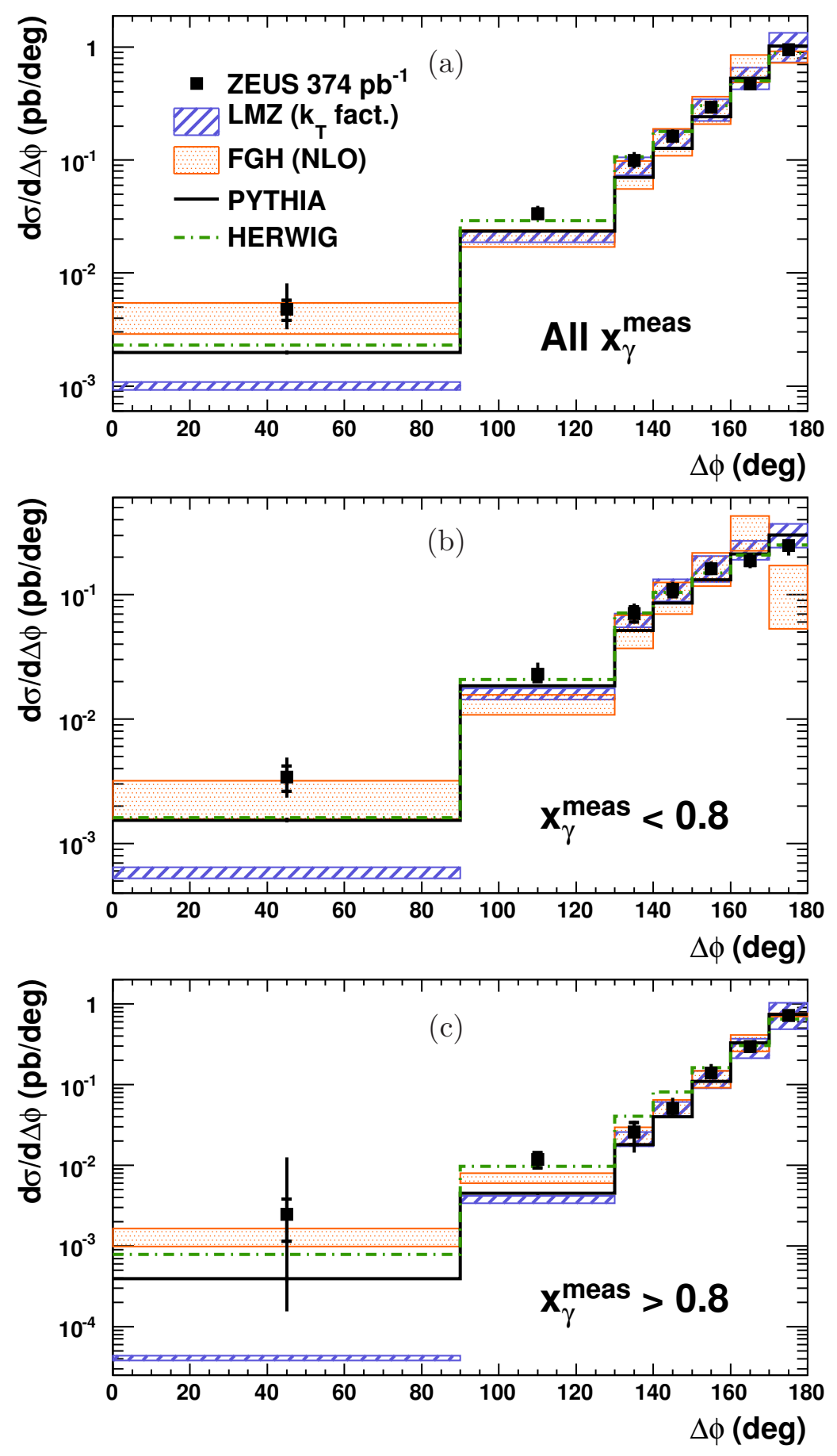

Figure 7. Differential cross sections as functions of $\Delta \phi$ for (a) all $x_{\gamma}^{\text {meas }}$, (b) $x_{\gamma}^{\text {meas }}>0.8$ (c) $x_{\gamma}^{\text {meas }}<0.8$ for events containing an isolated photon accompanied by a jet, compared to predictions from FGH, LMZ, PythiA and HeRWig. Other details as for figure 3. 


\begin{tabular}{|c|c|c|c|}
\hline \multicolumn{2}{|c|}{$\begin{array}{c}E_{T}^{\gamma} \text { range } \\
(\mathrm{GeV})\end{array}$} & $\frac{d \sigma}{d E_{T}^{\gamma}}\left(\mathrm{pb} \mathrm{GeV}^{-1}\right)$ & had. corr. \\
\hline \multicolumn{4}{|c|}{$x_{\gamma}^{\text {meas }}>0.8$} \\
\hline 6.0 & $\begin{array}{l}-\quad 7.0 \\
-\quad 1\end{array}$ & $3.79 \pm 0.26$ (stat.) ${ }_{-0.16}^{+0.37}$ (syst.) & 0.84 \\
\hline 7.0 & 8.5 & $2.60 \pm 0.17$ (stat.) ${ }_{-0.14}^{+0.23}$ (syst.) & 0.90 \\
\hline 8.5 & $-\quad 10.0$ & $1.55 \pm 0.13$ (stat.) ${ }_{-0.13}^{+0.21}$ (syst.) & 0.96 \\
\hline 10.0 & $-\quad 15.0$ & $0.63 \pm 0.04$ (stat.) ${ }_{-0.04}^{+0.04}$ (syst.) & 0.98 \\
\hline \multicolumn{4}{|c|}{$x_{\gamma}^{\text {meas }}<0.8$} \\
\hline 6.0 & 7.0 & $3.22 \pm 0.24$ (stat.) ${ }_{-0.28}^{+0.34}$ (syst.) & 0.79 \\
\hline 7.0 & 8.5 & $2.07 \pm 0.14$ (stat.) ${ }_{-0.16}^{+0.15}$ (syst.) & 0.80 \\
\hline 8.5 & $-\quad 10.0$ & $1.06 \pm 0.10$ (stat.) ${ }_{-0.15}^{+0.07}$ (syst.) & 0.81 \\
\hline 10.0 & $-\quad 15.0$ & $0.27 \pm 0.03$ (stat.) ${ }_{-0.03}^{+0.02}$ (syst.) & 0.83 \\
\hline \multicolumn{4}{|c|}{$x_{\gamma}^{\text {meas }}<0.7$} \\
\hline 6.0 & $-\quad 7.0$ & $2.37 \pm 0.21$ (stat.) ${ }_{-0.21}^{+0.29}$ (syst.) & 0.72 \\
\hline 7.0 & 8.5 & $1.32 \pm 0.12$ (stat.) ${ }_{-0.09}^{+0.10}$ (syst.) & 0.75 \\
\hline 8.5 & $\begin{array}{l}-\quad 10.0 \\
-1\end{array}$ & $0.66 \pm 0.09$ (stat.) ${ }_{-0.08}^{+0.07}$ (syst.) & 0.77 \\
\hline 10.0 & $-\quad 15.0$ & $0.18 \pm 0.03$ (stat.) ${ }_{-0.03}^{+0.02}$ (syst.) & 0.80 \\
\hline
\end{tabular}

Table 1. Differential cross-section $\frac{d \sigma}{d E_{T}^{\gamma}}$ for photons accompanied by a jet, and hadronisation correction.

\begin{tabular}{|c|c|c|c|}
\hline \multicolumn{2}{|c|}{$\eta^{\gamma}$ range } & $\frac{d \sigma}{d \eta^{\gamma}}(\mathrm{pb})$ & had. corr \\
\hline \multicolumn{4}{|c|}{$x_{\gamma}^{\text {meas }}>0.8$} \\
\hline-0.7 & $-\quad-0.3$ & $10.69 \pm 0.62$ (stat.) ${ }_{-0.71}^{+1.20}$ (syst.) & 0.93 \\
\hline-0.3 & 0.1 & $10.07 \pm 0.59$ (stat.) ${ }_{-0.63}^{+0.66}$ (syst.) & 0.93 \\
\hline 0.1 & 0.5 & $7.06 \pm 0.56(\text { stat. })_{-0.36}^{+0.51}$ (syst.) & 0.90 \\
\hline 0.5 & 0.9 & $4.00 \pm 0.50$ (stat.) ${ }_{-0.20}^{+0.36}$ (syst.) & 0.87 \\
\hline \multicolumn{4}{|c|}{$x_{\gamma}^{\text {meas }}<0.8$} \\
\hline-0.7 & $-\quad-0.3$ & $4.54 \pm 0.40$ (stat.) ${ }_{-0.42}^{+0.41}$ (syst.) & 0.84 \\
\hline-0.3 & 0.1 & $6.83 \pm 0.44$ (stat.) ${ }_{-0.49}^{+0.46}$ (syst.) & 0.80 \\
\hline 0.1 & 0.5 & $7.20 \pm 0.48$ (stat.) ${ }_{-0.68}^{+0.47}$ (syst.) & 0.80 \\
\hline 0.5 & 0.9 & $4.08 \pm 0.51$ (stat.) ${ }_{-0.21}^{+0.43}$ (syst.) & 0.79 \\
\hline \multicolumn{4}{|c|}{$x_{\gamma}^{\text {meas }}<0.7$} \\
\hline-0.7 & $-\quad-0.3$ & $2.79 \pm 0.31$ (stat.) ${ }_{-0.27}^{+0.26}$ (syst.) & 0.78 \\
\hline-0.3 & 0.1 & $4.56 \pm 0.38$ (stat.) ${ }_{-0.31}^{+0.31}$ (syst.) & 0.74 \\
\hline 0.1 & 0.5 & $5.12 \pm 0.44$ (stat.) ${ }_{-0.52}^{+0.32}$ (syst.) & 0.74 \\
\hline 0.5 & 0.9 & $3.15 \pm 0.49$ (stat.) ${ }_{-0.26}^{+0.43}$ (syst.) & 0.74 \\
\hline
\end{tabular}

Table 2. Differential cross-section $\frac{d \sigma}{d \eta^{\gamma}}$ for photons accompanied by a jet, and hadronisation correction. 


\begin{tabular}{|c|c|c|c|}
\hline \multicolumn{2}{|c|}{$\begin{array}{c}E_{T}^{\text {jet }} \text { range } \\
(\mathrm{GeV})\end{array}$} & $\frac{d \sigma}{d E_{T}^{\mathrm{jet}}}\left(\mathrm{pb} \mathrm{GeV}^{-1}\right)$ & had. corr. \\
\hline \multicolumn{4}{|c|}{$x_{\gamma}^{\text {meas }}>0.8$} \\
\hline 4.0 & -6.0 & $1.29 \pm 0.10$ (stat.) ${ }_{-0.16}^{+0.21}$ (syst.) & 0.81 \\
\hline 6.0 & 8.0 & $2.13 \pm 0.14$ (stat.) ${ }_{-0.15}^{+0.21}$ (syst.) & 0.83 \\
\hline 8.0 & $-\quad 10.0$ & $1.56 \pm 0.12$ (stat.) ${ }_{-0.14}^{+0.12}$ (syst.) & 0.96 \\
\hline 10.0 & $-\quad 15.0$ & $0.59 \pm 0.04$ (stat.) ${ }_{-0.05}^{+0.07}$ (syst.) & 1.05 \\
\hline \multicolumn{4}{|c|}{$x_{\gamma}^{\text {meas }}<0.8$} \\
\hline 4.0 & $\begin{array}{l}-6.0 \\
-\quad 6\end{array}$ & $1.43 \pm 0.10$ (stat.) ${ }_{-0.10}^{+0.17}$ (syst.) & 0.84 \\
\hline 6.0 & 8.0 & $1.29 \pm 0.10$ (stat.) ${ }_{-0.07}^{+0.08}$ (syst.) & 0.73 \\
\hline 8.0 & $-\quad 10.0$ & $1.06 \pm 0.09$ (stat.) ${ }_{-0.17}^{+0.10}$ (syst.) & 0.80 \\
\hline 10.0 & $-\quad 15.0$ & $0.28 \pm 0.03$ (stat.) ${ }_{-0.04}^{+0.02}$ (syst.) & 0.87 \\
\hline \multicolumn{4}{|c|}{$x_{\gamma}^{\text {meas }}<0.7$} \\
\hline 4.0 & $\begin{array}{l}-6.0 \\
-\quad 6.0\end{array}$ & $1.07 \pm 0.09$ (stat.) ${ }_{-0.08}^{+0.15}$ (syst.) & 0.76 \\
\hline 6.0 & 8.0 & $0.82 \pm 0.09$ (stat.) ${ }_{-0.05}^{+0.05}$ (syst.) & 0.68 \\
\hline 8.0 & $-\quad 10.0$ & $0.73 \pm 0.08$ (stat.) ${ }_{-0.14}^{+0.07}$ (syst.) & 0.77 \\
\hline 10.0 & $-\quad 15.0$ & $0.20 \pm 0.03$ (stat.) ${ }_{-0.03}^{+0.02}$ (syst.) & 0.83 \\
\hline
\end{tabular}

Table 3. Differential cross-section $\frac{d \sigma}{d E_{T}^{\text {jet }}}$ for photons accompanied by a jet, and hadronisation correction.

\begin{tabular}{|c|c|c|c|}
\hline \multicolumn{2}{|c|}{$\eta^{\text {jet }}$ range } & $\frac{d \sigma}{d \eta^{\text {jet }}}(\mathrm{pb})$ & had. corr. \\
\hline \multicolumn{4}{|c|}{$x_{\gamma}^{\text {meas }}>0.8$} \\
\hline-1.5 & $-\quad-0.7$ & $2.04 \pm 0.22$ (stat.) ${ }_{-0.18}^{+0.18}$ (syst.) & 0.68 \\
\hline-0.7 & 0.1 & $5.60 \pm 0.35$ (stat.) ${ }_{-0.18}^{+0.31}$ (syst.) & 0.83 \\
\hline 0.1 & 0.9 & $5.32 \pm 0.32$ (stat.) ${ }_{-0.32}^{+0.45}$ (syst.) & 1.09 \\
\hline 0.9 & 1.8 & $2.87 \pm 0.21$ (stat.) ${ }_{-0.23}^{+0.38}$ (syst.) & 1.33 \\
\hline \multicolumn{4}{|c|}{$x_{\gamma}^{\text {meas }}<0.8$} \\
\hline-1.5 & $-\quad-0.7$ & $0.43 \pm 0.10$ (stat.) ${ }_{-0.09}^{+0.07}$ (syst.) & 1.15 \\
\hline-0.7 & 0.1 & $2.22 \pm 0.21$ (stat.) ${ }_{-0.19}^{+0.25}$ (syst.) & 0.79 \\
\hline 0.1 & 0.9 & $4.29 \pm 0.26$ (stat.) ${ }_{-0.35}^{+0.31}$ (syst.) & 0.73 \\
\hline 0.9 & 1.8 & $3.94 \pm 0.27$ (stat.) ${ }_{-0.30}^{+0.24}$ (syst.) & 0.85 \\
\hline \multicolumn{4}{|c|}{$x_{\gamma}^{\text {meas }}<0.7$} \\
\hline-1.5 & $-\quad-0.7$ & $0.08 \pm 0.08$ (stat.) ${ }_{-0.05}^{+0.08}$ (syst.) & 0.83 \\
\hline-0.7 & 0.1 & $1.18 \pm 0.17$ (stat.) ${ }_{-0.08}^{+0.14}$ (syst.) & 0.69 \\
\hline 0.1 & 0.9 & $3.11 \pm 0.23$ (stat.) ${ }_{-0.26}^{+0.22}$ (syst.) & 0.69 \\
\hline 0.9 & 1.8 & $3.05 \pm 0.25$ (stat.) ${ }_{-0.24}^{+0.22}$ (syst.) & 0.82 \\
\hline
\end{tabular}

Table 4. Differential cross-section $\frac{d \sigma}{d \eta^{\text {jet }}}$ for photons accompanied by a jet, and hadronisation correction. 


\begin{tabular}{|c|c|c|c|}
\hline \multicolumn{2}{|c|}{$x_{p}^{\text {obs }}$ range } & $\frac{d \sigma}{d x_{p}^{\text {obs }}}(\mathrm{pb})$ & had. corr. \\
\hline \multicolumn{4}{|c|}{ All $x_{\gamma}^{\text {meas }}$} \\
\hline 0.0 & -0.005 & $297.6 \pm 30.4$ (stat.) ${ }_{-51.3}^{+46.0}$ (syst.) & 0.76 \\
\hline 0.005 & $-\quad 0.010$ & $1471.5 \pm 63.2$ (stat.) ${ }_{-124.3}^{+135.3}$ (syst.) & 0.80 \\
\hline 0.010 & -0.015 & $1160.0 \pm 57.5$ (stat.) ${ }_{-57.2}^{+56.9}$ (syst.) & 0.90 \\
\hline 0.015 & $-\quad 0.025$ & $514.5 \pm 27.8$ (stat.) ${ }_{-29.4}^{+20.5}$ (syst.) & 0.94 \\
\hline 0.025 & -0.040 & $130.1 \pm 11.7$ (stat.) ${ }_{-17.1}^{+6.6}$ (syst.) & 0.99 \\
\hline 0.040 & $-\quad 0.070$ & $12.6 \pm 2.6$ (stat.) ${ }_{-3.8}^{+1.0}$ (syst.) & 1.00 \\
\hline \multicolumn{4}{|c|}{$x_{\gamma}^{\text {meas }}>0.8$} \\
\hline 0.0 & -0.005 & $199.5 \pm 27.3$ (stat.) ${ }_{-15.3}^{+23.2}$ (syst.) & 0.72 \\
\hline 0.005 & -0.010 & $975.3 \pm 54.4$ (stat.) ${ }_{-68.1}^{+81.8}$ (syst.) & 0.82 \\
\hline 0.010 & -0.015 & $662.8 \pm 46.7$ (stat.) ${ }_{-28.8}^{+68.4}$ (syst.) & 1.00 \\
\hline 0.015 & -0.025 & $276.9 \pm 21.3$ (stat.) ${ }_{-15.3}^{+21.4}$ (syst.) & 1.12 \\
\hline 0.025 & $-\quad 0.040$ & $61.9 \pm 8.0$ (stat.) ${ }_{-4.3}^{+3.6}$ (syst.) & 1.26 \\
\hline 0.040 & $-\quad 0.070$ & $0.9 \pm 0.9$ (stat.) ${ }_{-0.6}^{+1.5}$ (syst.) & 1.29 \\
\hline \multicolumn{4}{|c|}{$x_{\gamma}^{\text {meas }}<0.8$} \\
\hline 0.0 & -0.005 & $79.6 \pm 14.8$ (stat.) ${ }_{-31.4}^{+20.7}$ (syst.) & 0.95 \\
\hline 0.005 & -0.010 & $492.3 \pm 37.0$ (stat.) ${ }_{-53.5}^{+52.3}$ (syst.) & 0.77 \\
\hline 0.010 & $-\quad 0.015$ & $515.2 \pm 38.1$ (stat.) ${ }_{-28.0}^{+24.7}$ (syst.) & 0.78 \\
\hline 0.015 & -0.025 & $249.9 \pm 20.6$ (stat.) ${ }_{-21.9}^{+14.0}$ (syst.) & 0.81 \\
\hline 0.025 & -0.040 & $70.9 \pm 9.4$ (stat.) ${ }_{-6.1}^{+3.7}$ (syst.) & 0.85 \\
\hline 0.040 & $-\quad 0.070$ & $5.3 \pm 2.2$ (stat.) $)_{-1.0}^{+0.9}$ (syst.) & 0.86 \\
\hline \multicolumn{4}{|c|}{$x_{\gamma}^{\text {meas }}<0.7$} \\
\hline 0.0 & -0.005 & $35.5 \pm 11.4$ (stat.) ${ }_{-10.4}^{+9.4}$ (syst.) & 0.69 \\
\hline 0.005 & -0.010 & $298.3 \pm 30.4$ (stat.) ${ }_{-39.5}^{+34.9}$ (syst.) & 0.68 \\
\hline 0.010 & -0.015 & $366.1 \pm 33.5$ (stat.) ${ }_{-21.6}^{+21.0}$ (syst.) & 0.73 \\
\hline 0.015 & -0.025 & $193.6 \pm 18.8$ (stat.) ${ }_{-21.6}^{+12.6}$ (syst.) & 0.78 \\
\hline 0.025 & -0.040 & $51.4 \pm 9.0$ (stat.) ${ }_{-4.8}^{+2.1}$ (syst.) & 0.83 \\
\hline 0.040 & $-\quad 0.070$ & $3.8 \pm 2.1$ (stat.) ${ }_{-0.9}^{+1.1}$ (syst.) & 0.82 \\
\hline
\end{tabular}

Table 5. Differential cross-section $\frac{d \sigma}{d x_{p}^{\text {obs }}}$ for photons accompanied by a jet, and hadronisation correction. 


\begin{tabular}{|c|c|c|c|c|}
\hline \multicolumn{3}{|c|}{$\left(\eta^{\gamma}-\eta^{\text {jet }}\right)$ range } & $\frac{d \sigma}{d\left(\eta^{\gamma}-\eta^{\mathrm{jet}}\right)}(\mathrm{pb})$ & had. corr. \\
\hline \multicolumn{5}{|c|}{ All $x_{\gamma}^{\text {meas }}$} \\
\hline-2.2 & & -1.5 & $3.17 \pm 0.24$ (stat.) ${ }_{-0.18}^{+0.14}$ (syst.) & 1.04 \\
\hline-1.5 & - & -0.8 & $6.56 \pm 0.35$ (stat.) ${ }_{-0.48}^{+0.31}$ (syst.) & 0.96 \\
\hline-0.8 & - & -0.1 & $8.57 \pm 0.40$ (stat.) ${ }_{-0.59}^{+0.58}$ (syst.) & 0.89 \\
\hline-0.1 & - & 0.6 & $7.42 \pm 0.38$ (stat.) ${ }_{-0.31}^{+0.52}$ (syst.) & 0.84 \\
\hline 0.6 & - & 1.3 & $3.99 \pm 0.32$ (stat.) ${ }_{-0.22}^{+0.23}$ (syst.) & 0.77 \\
\hline 1.3 & - & 2.0 & $0.98 \pm 0.19$ (stat.) ${ }_{-0.07}^{+0.14}$ (syst.) & 0.73 \\
\hline \multicolumn{5}{|c|}{$x_{\gamma}^{\text {meas }}>0.8$} \\
\hline-2.2 & - & -1.5 & $1.81 \pm 0.19$ (stat.) ${ }_{-0.15}^{+0.35}$ (syst.) & 1.32 \\
\hline-1.5 & - & -0.8 & $3.41 \pm 0.26$ (stat.) ${ }_{-0.23}^{+0.33}$ (syst.) & 1.18 \\
\hline-0.8 & - & -0.1 & $4.44 \pm 0.31$ (stat.) ${ }_{-0.27}^{+0.53}$ (syst.) & 1.04 \\
\hline-0.1 & - & 0.6 & $4.88 \pm 0.34$ (stat.) ${ }_{-0.21}^{+0.37}$ (syst.) & 0.88 \\
\hline 0.6 & - & 1.3 & $2.77 \pm 0.29$ (stat.) ${ }_{-0.18}^{+0.18}$ (syst.) & 0.74 \\
\hline 1.3 & - & 2.0 & $0.74 \pm 0.18$ (stat.) ${ }_{-0.09}^{+0.09}$ (syst.) & 0.65 \\
\hline \multicolumn{5}{|c|}{$x_{\gamma}^{\text {meas }}<0.8$} \\
\hline-2.2 & - & -1.5 & $1.49 \pm 0.17$ (stat.) ${ }_{-0.12}^{+0.08}$ (syst.) & 0.89 \\
\hline-1.5 & - & -0.8 & $3.34 \pm 0.27$ (stat.) ${ }_{-0.29}^{+0.23}$ (syst.) & 0.83 \\
\hline-0.8 & - & -0.1 & $4.23 \pm 0.29$ (stat.) ${ }_{-0.36}^{+0.34}$ (syst.) & 0.75 \\
\hline-0.1 & - & 0.6 & $2.63 \pm 0.24$ (stat.) ${ }_{-0.23}^{+0.24}$ (syst.) & 0.76 \\
\hline 0.6 & - & 1.3 & $1.24 \pm 0.18$ (stat.) $)_{-0.13}^{+0.10}$ (syst.) & 0.88 \\
\hline 1.3 & - & 2.0 & $0.19 \pm 0.09$ (stat.) ${ }_{-0.05}^{+0.07}$ (syst.) & 1.15 \\
\hline \multicolumn{5}{|c|}{$x_{\gamma}^{\text {meas }}<0.7$} \\
\hline-2.2 & - & -1.5 & $1.02 \pm 0.15$ (stat.) $)_{-0.08}^{+0.08}$ (syst.) & 0.85 \\
\hline-1.5 & - & -0.8 & $2.56 \pm 0.25$ (stat.) ${ }_{-0.25}^{+0.18}$ (syst.) & 0.81 \\
\hline-0.8 & - & -0.1 & $3.19 \pm 0.27$ (stat.) ${ }_{-0.27}^{+0.24}$ (syst.) & 0.72 \\
\hline-0.1 & - & 0.6 & $1.69 \pm 0.21$ (stat.) ${ }_{-0.11}^{+0.24}$ (syst.) & 0.68 \\
\hline 0.6 & - & 1.3 & $0.61 \pm 0.15$ (stat.) $)_{-0.10}^{+0.08}$ (syst.) & 0.71 \\
\hline 1.3 & - & 2.0 & $0.00 \pm 0.48$ (stat.) $)_{-0.00}^{+0.13}$ (syst.) & 0.87 \\
\hline
\end{tabular}

Table 6. Differential cross-section $\frac{d \sigma}{d\left(\eta^{\gamma}-\eta^{\text {jet }}\right)}$ for photons accompanied by a jet, and hadronisation correction. 


\begin{tabular}{|c|c|c|c|c|}
\hline \multicolumn{3}{|c|}{$\begin{array}{c}\Delta \phi \text { range } \\
(\text { deg. })\end{array}$} & $\frac{d \sigma}{d \Delta \phi}\left(\right.$ pb deg. $\left.^{-1}\right)$ & had. corr \\
\hline \multicolumn{5}{|c|}{ All $x_{\gamma}^{\text {meas }}$} \\
\hline & & 90.0 & $0.0048 \pm 0.0010$ (stat.) ${ }_{-0.0013}^{+0.0032}$ (syst.) & 0.78 \\
\hline 90.0 & - & 130.0 & $0.033 \pm 0.004$ (stat.) ${ }_{-0.002}^{+0.005}$ (syst.) & 0.81 \\
\hline 130.0 & - & 140.0 & $0.100 \pm 0.012$ (stat.) ${ }_{-0.009}^{+0.013}$ (syst.) & 0.82 \\
\hline 140.0 & - & 150.0 & $0.164 \pm 0.016$ (stat.) ${ }_{-0.014}^{+0.018}$ (syst.) & 0.85 \\
\hline 150.0 & - & 160.0 & $0.296 \pm 0.019$ (stat.) ${ }_{-0.016}^{+0.027}$ (syst.) & 0.86 \\
\hline 160.0 & - & 170.0 & $0.473 \pm 0.026$ (stat.) ${ }_{-0.026}^{+0.019}$ (syst.) & 0.89 \\
\hline 170.0 & - & 180.0 & $0.951 \pm 0.036$ (stat.) ${ }_{-0.066}^{+0.030}$ (syst.) & 0.86 \\
\hline \multicolumn{5}{|c|}{$x_{\gamma}^{\text {meas }}>0.8$} \\
\hline 0.0 & - & 90.0 & $0.002 \pm 0.001$ (stat.) ${ }_{-0.002}^{+0.010}$ (syst.) & 0.57 \\
\hline 90.0 & - & 130.0 & $0.012 \pm 0.003$ (stat.) ${ }_{-0.001}^{+0.001}$ (syst.) & 0.76 \\
\hline 130.0 & - & 140.0 & $0.026 \pm 0.008$ (stat.) ${ }_{-0.009}^{+0.005}$ (syst.) & 0.77 \\
\hline 140.0 & - & 150.0 & $0.051 \pm 0.010$ (stat.) ${ }_{-0.006}^{+0.015}$ (syst.) & 0.85 \\
\hline 150.0 & - & 160.0 & $0.140 \pm 0.014$ (stat.) ${ }_{-0.006}^{+0.037}$ (syst.) & 0.89 \\
\hline 160.0 & - & 170.0 & $0.295 \pm 0.022$ (stat.) ${ }_{-0.033}^{+0.014}$ (syst.) & 0.93 \\
\hline 170.0 & - & 180.0 & $0.720 \pm 0.034$ (stat.) ${ }_{-0.055}^{+0.045}$ (syst.) & 0.91 \\
\hline \multicolumn{5}{|c|}{$x_{\gamma}^{\text {meas }}<0.8$} \\
\hline 0.0 & - & 90.0 & $0.0034 \pm 0.0008$ (stat.) ${ }_{-0.0007}^{+0.0013}$ (syst.) & 0.79 \\
\hline 90.0 & - & 130.0 & $0.0230 \pm 0.0030$ (stat.) ${ }_{-0.0014}^{+0.0045}$ (syst.) & 0.82 \\
\hline 130.0 & - & 140.0 & $0.070 \pm 0.010$ (stat.) ${ }_{-0.007}^{+0.011}$ (syst.) & 0.84 \\
\hline 140.0 & - & 150.0 & $0.110 \pm 0.014$ (stat.) ${ }_{-0.008}^{+0.009}$ (syst.) & 0.86 \\
\hline 150.0 & - & 160.0 & $0.162 \pm 0.015$ (stat.) ${ }_{-0.009}^{+0.018}$ (syst.) & 0.84 \\
\hline 160.0 & - & 170.0 & $0.187 \pm 0.017$ (stat.) ${ }_{-0.017}^{+0.011}$ (syst.) & 0.82 \\
\hline 170.0 & - & 180.0 & $0.247 \pm 0.020$ (stat.) ${ }_{-0.035}^{+0.016}$ (syst.) & 0.76 \\
\hline \multicolumn{5}{|c|}{$x_{\gamma}^{\text {meas }}<0.7$} \\
\hline 0.0 & - & 90.0 & $0.0023 \pm 0.0006$ (stat.) ${ }_{-0.0005}^{+0.0010}$ (syst.) & 0.75 \\
\hline 90.0 & - & 130.0 & $0.0168 \pm 0.0027$ (stat.) ${ }_{-0.0015}^{+0.0051}$ (syst.) & 0.78 \\
\hline 130.0 & - & 140.0 & $0.046 \pm 0.008$ (stat.) ${ }_{-0.004}^{+0.006}$ (syst.) & 0.80 \\
\hline 140.0 & - & 150.0 & $0.063 \pm 0.012$ (stat.) ${ }_{-0.005}^{+0.016}$ (syst.) & 0.79 \\
\hline 150.0 & - & 160.0 & $0.104 \pm 0.013$ (stat.) ${ }_{-0.007}^{+0.007}$ (syst.) & 0.77 \\
\hline 160.0 & - & 170.0 & $0.133 \pm 0.015$ (stat.) ${ }_{-0.012}^{+0.008}$ (syst.) & 0.76 \\
\hline 170.0 & - & 180.0 & $0.172 \pm 0.017$ (stat.) ${ }_{-0.026}^{+0.010}$ (syst.) & 0.70 \\
\hline
\end{tabular}

Table 7. Differential cross-section $\frac{d \sigma}{d \Delta \phi}$ for photons accompanied by a jet, and hadronisation correction. 


\section{Acknowledgments}

We appreciate the contributions to the construction, maintenance and operation of the ZEUS detector made by many people who are not listed as authors. The HERA machine group and the DESY computing staff are especially acknowledged for their success in providing excellent operation of the collider and the data-analysis environment. We thank the DESY directorate for their strong support and encouragement. We also thank M. Fontannaz, G. Heinrich, A. Lipatov, M. Malyshev and N. Zotov for providing assistance and theoretical results.

Open Access. This article is distributed under the terms of the Creative Commons Attribution License (CC-BY 4.0), which permits any use, distribution and reproduction in any medium, provided the original author(s) and source are credited.

\section{References}

[1] ZEUS collaboration, H. Abramowicz et al., Photoproduction of Isolated Photons, Inclusively and with a Jet, at HERA, Phys. Lett. B 730 (2014) 293 [arXiv: 1312.1539] [inSPIRE].

[2] ZEUS collaboration, J. Breitweg et al., Observation of isolated high $E(t)$ photons in photoproduction at HERA, Phys. Lett. B 413 (1997) 201 [hep-ex/9708038] [INSPIRE].

[3] ZEUS collaboration, J. Breitweg et al., Measurement of inclusive prompt photon photoproduction at HERA, Phys. Lett. B 472 (2000) 175 [hep-ex/9910045] [inSPIRE].

[4] ZEUS collaboration, S. Chekanov et al., Study of the effective transverse momentum of partons in the proton using prompt photons in photoproduction at HERA,

Phys. Lett. B 511 (2001) 19 [hep-ex/0104001] [InSPIRE].

[5] ZEUS collaboration, S. Chekanov et al., Measurement of prompt photons with associated jets in photoproduction at HERA, Eur. Phys. J. C 49 (2007) 511 [hep-ex/0608028] [INSPIRE].

[6] H1 collaboration, A. Aktas et al., Measurement of prompt photon cross sections in photoproduction at HERA, Eur. Phys. J. C 38 (2005) 437 [hep-ex/0407018] [INSPIRE].

[7] H1 collaboration, F.D. Aaron et al., Prompt Photons in Photoproduction at HERA, Eur. Phys. J. C 66 (2010) 17 [arXiv:0910.5631] [INSPIRE].

[8] H1 collaboration, F.D. Aaron et al., Measurement of isolated photon production in deep-inelastic scattering at HERA, Eur. Phys. J. C 54 (2008) 371 [arXiv:0711.4578] [INSPIRE].

[9] ZEUS collaboration, S. Chekanov et al., Observation of isolated high $E(T)$ photons in deep inelastic scattering, Phys. Lett. B 595 (2004) 86 [hep-ex/0402019] [INSPIRE].

[10] ZEUS collaboration, S. Chekanov et al., Measurement of isolated photon production in deep inelastic ep scattering, Phys. Lett. B 687 (2010) 16 [arXiv:0909.4223] [INSPIRE].

[11] ZEUS collaboration, H. Abramowicz et al., Measurement of isolated photons accompanied by jets in deep inelastic ep scattering, Phys. Lett. B 715 (2012) 88 [arXiv:1206.2270] [INSPIRE].

[12] ZEUS collaboration, M. Derrick et al., Dijet angular distributions in direct and resolved photoproduction at HERA, Phys. Lett. B 384 (1996) 401 [hep-ex/9605009] [INSPIRE]. 
[13] M. Fontannaz, J.P. Guillet and G. Heinrich, Isolated prompt photon photoproduction at NLO, Eur. Phys. J. C 21 (2001) 303 [hep-ph/0105121] [INSPIRE].

[14] M. Fontannaz and G. Heinrich, Isolated photon + jet photoproduction as a tool to constrain the gluon distribution in the proton and the photon, Eur. Phys. J. C 34 (2004) 191 [hep-ph/0312009] [INSPIRE].

[15] M. Fontannaz and G. Heinrich, private communication (2013).

[16] A.V. Lipatov and N.P. Zotov, Prompt photon photoproduction at HERA in the $k_{T}$-factorization approach, Phys. Rev. D 72 (2005) 054002 [hep-ph/0506044] [INSPIRE].

[17] A.V. Lipatov and N.P. Zotov, Study of non-collinear parton dynamics in the prompt photon photoproduction at HERA, Phys. Rev. D 81 (2010) 094027 [arXiv:0907.3303] [INSPIRE].

[18] A.V. Lipatov, M.A. Malyshev and N.P. Zotov, An extended study of the prompt photon photoproduction at HERA with $k_{T}$-factorization, Phys. Rev. D 88 (2013) 074001 [arXiv: 1307.3644] [INSPIRE].

[19] A.V. Lipatov, M.A. Malyshev and N.P. Zotov, private communication (2014).

[20] ZEUS collaboration, U. Holm eds., The ZEUS Detector, Status Report (unpublished), DESY (1993), available on http://www-zeus.desy.de/bluebook/bluebook.html.

[21] N. Harnew et al., Vertex Triggering Using Time Difference Measurements in the ZEUS Central Tracking Detector, Nucl. Instrum. Meth. A 279 (1989) 290 [INSPIRE].

[22] B. Foster et al., The Performance of the Zeus central tracking detector $z$-by-timing electronics in a transputer based data acquisition system, Nucl. Phys. Proc. Suppl. B 32 (1993) 181.

[23] ZEUS collaboration, B. Foster et al., The Design and construction of the ZEUS central tracking detector, Nucl. Instrum. Meth. A 338 (1994) 254 [INSPIRE].

[24] ZEUS collaboration, A. Polini et al., The design and performance of the ZEUS Micro Vertex detector, Nucl. Instrum. Meth. A 581 (2007) 656 [arXiv:0708.3011] [INSPIRE].

[25] M. Derrick et al., Design and construction of the ZEUS barrel calorimeter., Nucl. Instrum. Meth. A 309 (1991) 77 [INSPIRE].

[26] ZEUS Calorimeter Group, ZEUS collaboration, A. Andresen et al., Construction and beam test of the ZEUS forward and rear calorimeter, Nucl. Instrum. Meth. A 309 (1991) 101 [INSPIRE].

[27] A. Caldwell et al., Design and implementation of a high precision readout system for the ZEUS calorimeter, Nucl. Instrum. Meth. A 321 (1992) 356 [INSPIRE].

[28] Zeus Barrel Calorimeter Group collaboration, A. Bernstein et al., Beam tests of the ZEUS barrel calorimeter, Nucl. Instrum. Meth. A 336 (1993) 23 [INSPIRE].

[29] ZEUS collaboration, L. Adamczyk et al., Measurement of the Luminosity in the ZEUS Experiment at HERA II, Nucl. Instrum. Meth. A 744 (2014) 80 [arXiv:1306.1391] [INSPIRE].

[30] J. Andruszków et al., First measurement of HERA luminosity by ZEUS lumi monitor, Preprint DESY-92-066, DESY (1992).

[31] ZEUS collaboration, M. Derrick et al., Measurement of total and partial photon proton cross-sections at 180-GeV center-of-mass energy, Z. Phys. C 63 (1994) 391 [InSPIRE]. 
[32] ZEUS Luminosity Group collaboration, J. Andruszków et al., Luminosity measurement in the ZEUS experiment, Acta Phys. Polon. B 32 (2001) 2025 [InSPIRE].

[33] M. Helbich et al., The Spectrometer system for measuring ZEUS luminosity at HERA, Nucl. Instrum. Meth. A 565 (2006) 572 [physics/0512153] [INSPIRE].

[34] L. Bourhis, M. Fontannaz, J.P. Guillet and M. Werlen, Next-to-leading order determination of fragmentation functions, Eur. Phys. J. C 19 (2001) 89 [hep-ph/0009101] [InSPIRE].

[35] J. Pumplin, A. Belyaev, J. Huston, D. Stump and W.K. Tung, Parton distributions and the strong coupling: CTEQ6AB PDFs, JHEP 02 (2006) 032 [hep-ph/0512167] [INSPIRE].

[36] P. Aurenche, M. Fontannaz and J.P. Guillet, New NLO parametrizations of the parton distributions in real photons, Eur. Phys. J. C 44 (2005) 395 [hep-ph/0503259] [INSPIRE].

[37] M.A. Kimber, A.D. Martin and M.G. Ryskin, Unintegrated parton distributions, Phys. Rev. D 63 (2001) 114027 [hep-ph/0101348] [INSPIRE].

[38] G. Watt, A.D. Martin and M.G. Ryskin, Unintegrated parton distributions and inclusive jet production at HERA, Eur. Phys. J. C 31 (2003) 73 [hep-ph/0306169] [INSPIRE].

[39] A.D. Martin, W.J. Stirling, R.S. Thorne and G. Watt, Parton distributions for the LHC, Eur. Phys. J. C 63 (2009) 189 [arXiv:0901.0002] [inSPIRE].

[40] T. Sjöstrand et al., PYTHIA 6.4 physics and manual, JHEP 05 (2006) 026 [hep-ph/0603175] [INSPIRE].

[41] H.L. Lai et al., Improved parton distributions from global analysis of recent deep inelastic scattering and inclusive jet data, Phys. Rev. D 55 (1997) 1280 [hep-ph/9606399] [INSPIRE].

[42] M. Glück, E. Reya and A. Vogt, Parton structure of the photon beyond the leading order, Phys. Rev. D 45 (1992) 3986 [INSPIRE].

[43] M. Glück, E. Reya and A. Vogt, Photonic parton distributions, Phys. Rev. D 46 (1992) 1973 [INSPIRE].

[44] G. Corcella et al., HERWIG 6: An Event generator for hadron emission reactions with interfering gluons (including supersymmetric processes), JHEP 01 (2001) 010 [hep-ph/0011363] [INSPIRE].

[45] R. Brun et al., Geant3, Technical Report CERN-DD/EE/84-1, CERN, Geneva Switzerland (1987).

[46] W.H. Smith, K. Tokushuku and L.W. Wiggers, The ZEUS trigger system, in Proceedings of Computing in High-Energy Physics (CHEP), Annecy France (1992), C. Verkerk and W. Wojcik eds., pg. 222. Also in preprint DESY 92-150B.

[47] P.D. Allfrey et al., The design and performance of the ZEUS global tracking trigger, Nucl. Instrum. Meth. A 580 (2007) 1257 [INSPIRE].

[48] ZEUS collaboration, M. Derrick et al., Initial study of deep inelastic scattering with ZEUS at HERA, Phys. Lett. B 303 (1993) 183 [InSPIRE].

[49] ZEUS collaboration, J. Breitweg et al., Measurement of the diffractive structure function F2(D(4)) at HERA, Eur. Phys. J. C 1 (1998) 81 [hep-ex/9709021] [INSPIRE].

[50] ZEUS collaboration, J. Breitweg et al., Measurement of the diffractive cross-section in deep inelastic scattering using ZEUS 1994 data, Eur. Phys. J. C 6 (1999) 43 [hep-ex/9807010] [INSPIRE]. 
[51] S. Catani, Y.L. Dokshitzer, M.H. Seymour and B.R. Webber, Longitudinally invariant $K_{t}$ clustering algorithms for hadron hadron collisions, Nucl. Phys. B 406 (1993) 187 [INSPIRE].

[52] S.D. Ellis and D.E. Soper, Successive combination jet algorithm for hadron collisions, Phys. Rev. D 48 (1993) 3160 [hep-ph/9305266] [INSPIRE].

[53] ZEUS collaboration, S. Chekanov et al., Inclusive jet cross sections and dijet correlations in $D^{* \pm}$ photoproduction at HERA, Nucl. Phys. B 729 (2005) 492 [hep-ex/0507089] [INSPIRE].

[54] ZEUS collaboration, S. Chekanov et al., High-E(T) dijet photoproduction at HERA, Phys. Rev. D 76 (2007) 072011 [arXiv: 0706. 3809] [INSPIRE].

[55] ATLAS collaboration, Dynamics of isolated-photon plus jet production in pp collisions at $\sqrt{s}=7$ TeV with the ATLAS detector, Nucl. Phys. B 875 (2013) 483 [arXiv:1307.6795] [INSPIRE]. 


\section{The ZEUS collaboration}

H. Abramowicz ${ }^{27, u}$, I. Abt ${ }^{21}$, L. Adamczyk ${ }^{8}$, M. Adamus ${ }^{34}$, R. Aggarwal ${ }^{4, a}$, S. Antonelli ${ }^{2}$, O. Arslan $^{3}$, V. Aushev ${ }^{16,17, o}$, Y. Aushev ${ }^{17, o, p}$, O. Bachynska ${ }^{10}$, A.N. Barakbaev ${ }^{15}$, N. Bartosik ${ }^{10}$, O. Behnke ${ }^{10}$, J. Behr ${ }^{10}$, U. Behrens ${ }^{10}$, A. Bertolin ${ }^{23}$, S. Bhadra ${ }^{36}$, I. Bloch ${ }^{11}$, V. Bokhonov ${ }^{16, o}$, E.G. Boos $^{15}$, K. Borras $^{10}$, I. Brock ${ }^{3}$, R. Brugnera ${ }^{24}$, A. Bruni ${ }^{1}$, B. Brzozowska ${ }^{33}$, P.J. Bussey ${ }^{12}$, A. Caldwell ${ }^{21}$, M. Capua ${ }^{5}$, C.D. Catterall ${ }^{36}$, J. Chwastowski ${ }^{7, d}$, J. Ciborowski ${ }^{33, x}$,

R. Ciesielski ${ }^{10, f}$, A.M. Cooper-Sarkar ${ }^{22}$, M. Corradi ${ }^{1}$, F. Corriveau ${ }^{18}$, G. D'Agostini ${ }^{26}$, R.K. Dementiev ${ }^{20}$, R.C.E. Devenish ${ }^{22}$, G. Dolinska ${ }^{10}$, V. Drugakov ${ }^{11}$, S. Dusini ${ }^{23}$, J. Ferrando ${ }^{12}$, J. Figiel ${ }^{7}$, B. Foster ${ }^{13, l}$, G. Gach ${ }^{8}$, A. Garfagnini ${ }^{24}$, A. Geiser ${ }^{10}$, A. Gizhko ${ }^{10}$, L.K. Gladilin ${ }^{20}$, O. Gogota ${ }^{17}$, Yu.A. Golubkov ${ }^{20}$, J. Grebenyuk ${ }^{10}$, I. Gregor ${ }^{10}$, G. Grzelak ${ }^{33}$, O. Gueta ${ }^{27}$,

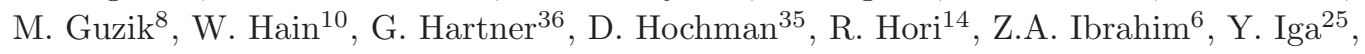
M. Ishitsuka ${ }^{28}$, A. Iudin ${ }^{17, p}$, F. Januschek ${ }^{10}$, I. Kadenko ${ }^{17}$, S. Kananov ${ }^{27}$, T. Kanno ${ }^{28}$, U. Karshon ${ }^{35}$, M. Kaur ${ }^{4}$, P. Kaur ${ }^{4, a}$, L.A. Khein ${ }^{20}$, D. Kisielewska ${ }^{8}$, R. Klanner ${ }^{13}$, U. Klein ${ }^{10, g}$, N. Kondrashova ${ }^{17, q}$, O. Kononenko ${ }^{17}$, Ie. $K_{\text {Korol }}{ }^{10}$, I.A. Korzhavina ${ }^{20}$, A. Kotański ${ }^{9}$, U. Kötz ${ }^{10}$, N. Kovalchuk ${ }^{17, r}$, H. Kowalski ${ }^{10}$, O. Kuprash ${ }^{10}$, M. Kuze ${ }^{28}$, B.B. Levchenko ${ }^{20}$, A. Levy ${ }^{27}$, V. Libov ${ }^{10}$, S. Limentani ${ }^{24}$, M. Lisovyi ${ }^{10}$, E. Lobodzinska ${ }^{10}$, W. Lohmann ${ }^{11}$, B. Löhr ${ }^{10}$, E. Lohrmann ${ }^{13}$, A. Longhin ${ }^{23, t}$, D. Lontkovskyi ${ }^{10}$, O.Yu. Lukina ${ }^{20}$, J. Maeda ${ }^{28, v}$, I. Makarenko ${ }^{10}$, J. Malka ${ }^{10}$, J.F. Martin ${ }^{31}$, S. Mergelmeyer ${ }^{3}$, F. Mohamad Idris ${ }^{6, c}$, K. Mujkic ${ }^{10, h}$,

V. Myronenko ${ }^{10, i}$, K. Nagano ${ }^{14}$, A. Nigro ${ }^{26}$, T. Nobe ${ }^{28}$, D. Notz ${ }^{10}$, R.J. Nowak ${ }^{33}$, K. Olkiewicz ${ }^{7}$, Yu. Onishchuk ${ }^{17}$, E. Paul ${ }^{3}$, W. Perlański ${ }^{33, y}$, H. Perrey ${ }^{10}$, N.S. Pokrovskiy ${ }^{15}$,

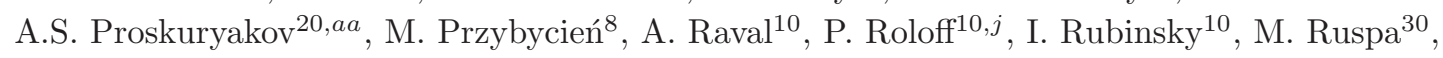
V. Samojlov ${ }^{15}$, D.H. Saxon ${ }^{12}$, M. Schioppa ${ }^{5}$, W.B. Schmidke ${ }^{21, s}$, U. Schneekloth ${ }^{10}$,

T. Schörner-Sadenius ${ }^{10}$, J. Schwartz ${ }^{18}$, L.M. Shcheglova ${ }^{20}$, R. Shevchenko ${ }^{17, p}$, O. Shkola ${ }^{17, r}$,

I. Singh ${ }^{4, b}$, I.O. Skillicorn ${ }^{12}$, W. Słomiński ${ }^{9, e}$, V. Sola ${ }^{13}$, A. Solano ${ }^{29}$, A. Spiridonov ${ }^{10, k}$,

L. Stanco ${ }^{23}$, N. Stefaniuk ${ }^{10}$, A. Stern ${ }^{27}$, T.P. Stewart ${ }^{31}$, P. Stopa ${ }^{7}$, J. Sztuk-Dambietz ${ }^{13}$,

D. Szuba ${ }^{13}$, J. Szuba ${ }^{10}$, E. Tassi ${ }^{5}$, T. Temiraliev ${ }^{15}$, K. Tokushuku ${ }^{14, m}$, J. Tomaszewska ${ }^{33, z}$,

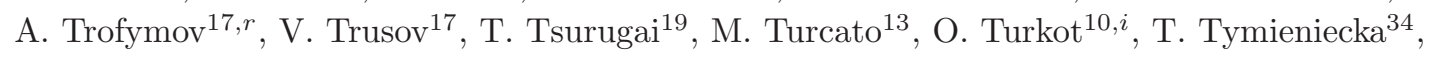

A. Verbytskyi ${ }^{21}$, O. Viazlo ${ }^{17}$, R. Walczak ${ }^{22}$, W.A.T. Wan Abdullah ${ }^{6}$, K. Wichmann ${ }^{10, i}$,

M. Wing ${ }^{32, w}$, G. Wolf ${ }^{10}$, S. Yamada ${ }^{14}$, Y. Yamazaki ${ }^{14, n}$, N. Zakharchuk ${ }^{17, r}$, A.F. Żarnecki ${ }^{33}$, L. Zawiejski ${ }^{7}$, O. Zenaiev ${ }^{10}$, B.O. Zhautykov ${ }^{15}$, N. Zhmak ${ }^{16, o}$, D.S. Zotkin ${ }^{20}$

1 INFN Bologna, Bologna, Italy ${ }^{A}$

2 University and INFN Bologna, Bologna, Italy ${ }^{A}$

3 Physikalisches Institut der Universität Bonn, Bonn, Germany ${ }^{B}$

4 Panjab University, Department of Physics, Chandigarh, India

${ }_{5}$ Calabria University, Physics Department and INFN, Cosenza, Italy ${ }^{A}$

6 National Centre for Particle Physics, Universiti Malaya, 50603 Kuala Lumpur, Malaysia ${ }^{C}$

7 The Henryk Niewodniczanski Institute of Nuclear Physics, Polish Academy of Sciences, Krakow, Poland ${ }^{D}$

8 AGH-University of Science and Technology, Faculty of Physics and Applied Computer Science, Krakow, Poland ${ }^{D}$

9 Department of Physics, Jagellonian University, Cracow, Poland

10 Deutsches Elektronen-Synchrotron DESY, Hamburg, Germany

11 Deutsches Elektronen-Synchrotron DESY, Zeuthen, Germany

12 School of Physics and Astronomy, University of Glasgow, Glasgow, United Kingdom ${ }^{E}$

13 Hamburg University, Institute of Experimental Physics, Hamburg, Germany F

14 Institute of Particle and Nuclear Studies, KEK, Tsukuba, Japan ${ }^{G}$

15 Institute of Physics and Technology of Ministry of Education and Science of Kazakhstan, Almaty, Kazakhstan 
Institute for Nuclear Research, National Academy of Sciences, Kyiv, Ukraine

17 Department of Nuclear Physics, National Taras Shevchenko University of Kyiv, Kyiv, Ukraine

18 Department of Physics, McGill University, Montréal, Québec, Canada H3A 2T8 ${ }^{H}$

19 Meiji Gakuin University, Faculty of General Education, Yokohama, Japan ${ }^{G}$

20 Lomonosov Moscow State University, Skobeltsyn Institute of Nuclear Physics, Moscow, Russia ${ }^{I}$

21 Max-Planck-Institut für Physik, München, Germany

22 Department of Physics, University of Oxford, Oxford, United Kingdom ${ }^{E}$

${ }^{23}$ INFN Padova, Padova, Italy ${ }^{A}$

24 Dipartimento di Fisica e Astronomia dell' Università and INFN, Padova, Italy ${ }^{A}$

25 Polytechnic University, Tokyo, Japan ${ }^{G}$

26 Dipartimento di Fisica, Università 'La Sapienza' and INFN, Rome, Italy ${ }^{A}$

27 Raymond and Beverly Sackler Faculty of Exact Sciences, School of Physics, Tel Aviv University, Tel Aviv, Israel ${ }^{J}$

28 Department of Physics, Tokyo Institute of Technology, Tokyo, Japan ${ }^{G}$

29 Università di Torino and INFN, Torino, Italy ${ }^{A}$

30 Università del Piemonte Orientale, Novara, and INFN, Torino, Italy ${ }^{A}$

31 Department of Physics, University of Toronto, Toronto, Ontario, Canada M5S $1 \mathrm{~A}^{\mathrm{H}}$

32 Physics and Astronomy Department, University College London, London, United Kingdom ${ }^{E}$

33 Faculty of Physics, University of Warsaw, Warsaw, Poland

34 National Centre for Nuclear Research, Warsaw, Poland

35 Department of Particle Physics and Astrophysics, Weizmann Institute, Rehovot, Israel

36 Department of Physics, York University, Ontario, Canada M3J 1 P3 ${ }^{H}$

A supported by the Italian National Institute for Nuclear Physics (INFN)

$B$ supported by the German Federal Ministry for Education and Research (BMBF), under contract No. 05 HO9PDF

C supported by HIR grant UM.C/625/1/HIR/149 and UMRG grants RU006-2013, RP012A-13AFR and RP012B-13AFR from Universiti Malaya, and ERGS grant ER004-2012A from the Ministry of Education, Malaysia

$D$ supported by the National Science Centre under contract No. DEC-2012/06/M/ST2/00428

E supported by the Science and Technology Facilities Council, U.K.

$F$ supported by the German Federal Ministry for Education and Research (BMBF), under contract No. 05h09GUF, and the SFB 676 of the Deutsche Forschungsgemeinschaft (DFG)

$G$ supported by the Japanese Ministry of Education, Culture, Sports, Science and Technology (MEXT) and its grants for Scientific Research

${ }^{H}$ supported by the Natural Sciences and Engineering Research Council of Canada (NSERC)

I supported by RF Presidential grant N 3042.2014.2 for the Leading Scientific Schools and by the Russian Ministry of Education and Science through its grant for Scientific Research on High Energy Physics

J supported by the Israel Science Foundation

a also funded by Max Planck Institute for Physics, Munich, Germany

${ }^{b}$ also funded by Max Planck Institute for Physics, Munich, Germany, now at Sri Guru Granth Sahib World University, Fatehgarh Sahib

$c$ also at Agensi Nuklear Malaysia, 43000 Kajang, Bangi, Malaysia

$d$ also at Cracow University of Technology, Faculty of Physics, Mathematics and Applied Computer Science, Poland

e partially supported by the Polish National Science Centre projects DEC-2011/01/B/ST2/03643 and DEC-2011/03/B/ST2/00220

$f$ now at Rockefeller University, New York, NY 10065, U.S.A.

$g$ now at University of Liverpool, United Kingdom

$h$ also affiliated with University College London, U.K. 
i supported by the Alexander von Humboldt Foundation

${ }^{j}$ now at CERN, Geneva, Switzerland

${ }^{k}$ also at Institute of Theoretical and Experimental Physics, Moscow, Russia

${ }^{l}$ Alexander von Humboldt Professor; also at DESY and University of Oxford

$m$ also at University of Tokyo, Japan

$n$ now at Kobe University, Japan

o supported by DESY, Germany

$p$ member of National Technical University of Ukraine, Kyiv Polytechnic Institute, Kyiv, Ukraine

$q$ now at DESY ATLAS group

$r$ member of National University of Kyiv - Mohyla Academy, Kyiv, Ukraine

$s$ now at BNL, U.S.A.

${ }^{t}$ now at LNF, Frascati, Italy

u also at Max Planck Institute for Physics, Munich, Germany, External Scientific Member

$v$ now at Tokyo Metropolitan University, Japan

$w$ also supported by DESY

$x$ also at Eódź University, Poland

y member of Eódź University, Poland

$z$ now at Polish Air Force Academy in Deblin

a deceased 